\title{
Theranostics
}

Review

$2011 ; 1: 83-101$

\section{Integrin Targeted MR Imaging}

\author{
Mingqian Tan and Zheng-Rong Lu ${ }^{凶}$ \\ Department of Biomedical Engineering, Case Western Reserve University, Cleveland, OH 44106, USA
}

Corresponding author: Dr. Zheng-Rong Lu, Wickenden 427, Mail Stop 7207, 10900 Euclid Avenue, Cleveland, OH 44106,USA. Tel: 216-368-0187; Email:zx1125@case.edu

(C) Ivyspring International Publisher. This is an open-access article distributed under the terms of the Creative Commons License (http://creativecommons.org/ licenses/by-nc-nd/3.0/). Reproduction is permitted for personal, noncommercial use, provided that the article is in whole, unmodified, and properly cited.

Published: 2011.01 .19

\begin{abstract}
Magnetic resonance imaging $(\mathrm{MRI})$ is a powerful medical diagnostic imaging modality for integrin targeted imaging, which uses the magnetic resonance of tissue water protons to display tissue anatomic structures with high spatial resolution. Contrast agents are often used in MRI to highlight specific regions of the body and make them easier to visualize. There are four main classes of MRI contrast agents based on their different contrast mechanisms, including $T_{1}, T_{2}$, chemical exchange saturation transfer (CEST) agents, and heteronuclear contrast agents. Integrins are an important family of heterodimeric transmembrane glycoproteins that function as mediators of cell-cell and cell-extracellular matrix interactions. The overexpressed integrins can be used as the molecular targets for designing suitable integrin targeted contrast agents for MR molecular imaging. Integrin targeted contrast agent includes a targeting agent specific to a target integrin, a paramagnetic agent and a linker connecting the targeting agent with the paramagnetic agent. Proper selection of targeting agents is critical for targeted MRI contrast agents to effectively bind to integrins for in vivo imaging. An ideal integrin targeted $M R$ contrast agent should be non-toxic, provide strong contrast enhancement at the target sites and can be completely excreted from the body after MR imaging. An overview of integrin targeted MR contrast agents based on small molecular and macromolecular Gd(III) complexes, lipid nanoparticles and superparamagnetic nanoparticles is provided for MR molecular imaging. By using proper delivery systems for loading sufficient Gd(III) chelates or superparamagnetic nanoparticles, effective molecular imaging of integrins with MRI has been demonstrated in animal models.
\end{abstract}

Key words: Magnetic resonance imaging (MRI); Gd(III) chelates; Superparamagnetic iron oxide nanoparticles; Integrin; RGD peptides.

\section{Introduction}

The benefits of earlier detection of life-threatening diseases have promoted the shift of biomedical research into the realm of predictive and preventative medicine. Molecular imaging of disease related biomarkers, such as over-expressed integrins, provides valuable information for earlier disease diagnosis and evaluation of treatment response. Magnetic resonance imaging (MRI) is a powerful medical diagnostic imaging modality for soft tissue imaging. It does not employ ionizing radiation and provides excellent whole-body soft tissue contrast with sub-millimeter resolution [1]. Contrast enhanced MRI has the potential to detect the disease related biomarkers in correlation to their accurate anatomical localization. With proper targeted MR contrast agents, MRI can elucidate the molecular mechanisms responsible for disease development and progression [2].

Integrins are a family of adhesion molecules through which cells can attach to extracellular matrices, to each other's surface or to different cell types [3-4]. All integrins are composed of a transmembrane-type heterodimer that consist of an a chain and a $\beta$ chain. Many physiological processes, including cell activation, migration, proliferation and differen- 
tiation, require direct contact between cells and the extracellular matrix [4]. Integrins play key roles in the regulation of normal and tumor cell migration and survival. For example, $\alpha_{v} \beta_{3}$ and $\alpha_{5} \beta_{1}$-integrins have been shown to regulate angiogenesis, the formation of new vessels, which contributes to wound healing and pathological development of solid tumor cancers [5-6]. Integrins have direct effects in preventing apoptosis in cancer cells and mediating proangiogenic interactions between endothelial cells and extracellular matrix [7]. With such diverse roles in cell biology, integrins have become attractive targets for the development of integrin-based diagnostic agents and therapeutic drugs for cancer treatment [4, 8-9].

The primary purpose of this review is to provide an overview of targeted MR imaging of integrins, which begins with an explanation of the basic principles of MRI, followed by a short introduction of MR contrast agents, and then a preview of the design and development of integrin binding MRI contrast agents as well as their application in MRI.

\section{Magnetic resonance imaging}

MRI is a powerful diagnostic modality that uses the magnetic resonance of tissue water protons to display tissue anatomic structures with high spatial resolution. The first MR image was published in 1973 by Paul Lauterbur [10]. The first studies performed on humans were published in 1977 [11] and the first publication of using paramagneic MR contrast agents appeared in the early 1980s [12]. During the past few decades, the applications of MRI in clinical oncology have been rapidly expanding. Compared with other imaging modalities, such as positron-emission tomography (PET), single-photon emission computed tomography (SPECT) and X-ray computed tomography (CT), MRI is advantageous because it does not require radioactive tracers or expose patients to harmful ionizing radiation [13-15].

\section{Principles of MRI}

Magnetic resonance imaging generates tissue images by measuring the interaction between an external magnetic field and the magnetic moment of water protons. A normal human adult has approximately $60 \%$ of the body weight as water. Water protons have a magnetic moment and the orientations of the magnetic moments are random in the absence of an external magnetic field [16]. When a patient is placed in a MRI scanner, the proton magnetic moments align either along (low energy) or against (high energy) the static magnetic field $\left(\mathrm{B}_{0}\right)$ of the scanner and create a net magnetization pointing in the direction of the main magnetic field of the scanner. The number of protons in the lower energy state is slightly higher than those in the higher energy field and the distribution is given by the Boltzmann equation (Eq. $1)$.

$$
\frac{N_{\uparrow}}{N_{\downarrow}}=e^{-\gamma h B_{0} / k_{B} T}
$$

where $N_{\uparrow}$ and $N_{\downarrow}$ refer to the lower and higher energy states of the protons, $\gamma$ is the proton gyromagnetic ratio, $B_{0}$ is the strength of the external magnetic field, $h$ is the Planck's constant, $6.626 \times 10^{-34} \mathrm{~J} \cdot \mathrm{s}$, $k_{B}$ is the Boltzmann's constant, $1.381 \times 10^{-23} \mathrm{~J} \cdot \mathrm{K}^{-1}$ and $T$ is the system temperature. The greater number of protons aligning with the external field creates a net magnetization $\left(\mathrm{M}_{0}\right)$, which consists of $\mathrm{M}_{Z}$ and $\mathrm{M}_{\mathrm{X}-\mathrm{Y}}$ components. This phenomenon is shown in Figure $\mathbf{1}$. The magnitude of the magnetization $\left(\mathrm{M}_{0}\right)$ is proportional to the external magnetic field strength, $\mathrm{B}_{0}$ : [17]

$$
\mathrm{M}_{0}=\chi \mathrm{B}_{0}
$$

where $X$ is known as the bulk magnetic susceptibility.

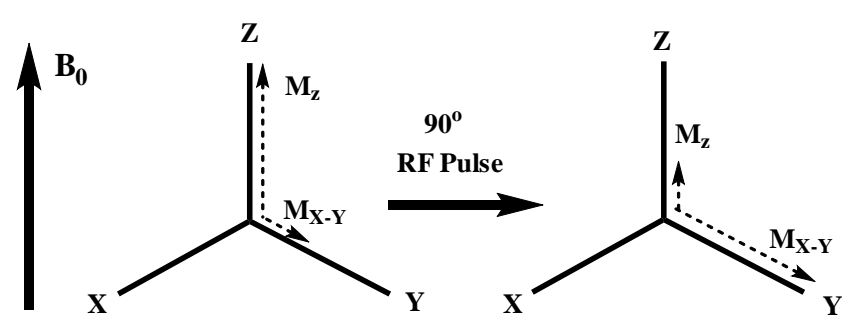

Figure I. Schematic illustration of the change of net magnetization vector immediately after a radio-frequency pulse. Adapted from reference [17] with permission.

Nuclear magnetic resonance (NMR) measures the change of the magnetization by applying radiofrequency $(\mathrm{RF})$ pulses. When an RF pulse is applied to create an oscillating electromagnetic field $\left(B_{1}\right)$ perpendicular to the main field, the protons absorb the energy and are temporarily excited to a higher energy state. The net magnetization rotates away from its equilibrium orientation due to the absorption of RF energy. After the radio frequency pulse is switched off, the absorbed energy is released to the surroundings by two simultaneous events: 1) the recovery of the net magnetization toward the equilibrium orientation, and 2) the dephase of the transverse magnetization $\mathrm{M}_{\mathrm{X}-\mathrm{Y}}$. The first case is termed as the spin-lattice or longitudinal $\left(\mathrm{T}_{1}\right)$ relaxation and the latter is spin-spin or transverse $\left(\mathrm{T}_{2}\right)$ relaxation. 
The rate of the relaxations is governed by the relaxation times. The $T_{1}$ relaxation time is defined as the time required after excitation for $63 \%$ of the longitudinal magnetization to recover to its original magnitude (Fig. 2). The process of $\mathrm{T}_{1}$ relaxation is described by equation 3 ,

$$
\mathrm{M}_{\mathrm{Z}}=\mathrm{M}_{0}\left(1-e^{-\tau / \mathrm{T}_{1}}\right)
$$

where $\mathrm{M}_{\mathrm{z}}$ is the longitudinal magnetization, $\mathrm{M}_{0}$ is the equilibrium magnetization, $\mathrm{T}_{1}$ is the longitudinal relaxation time and $\tau$ is the time following a radio frequency pulse.

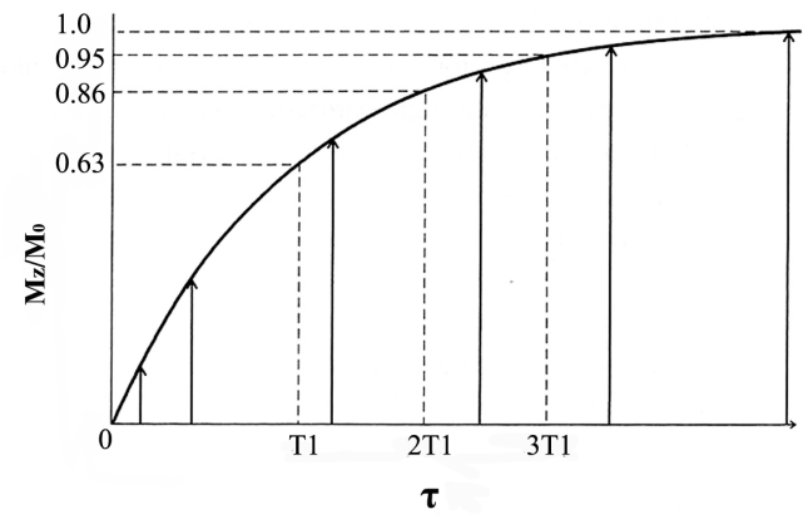

Figure 2. $T$, relaxation curve. The regrowth of longitudinal component of magnetization from the initial value $M_{z}(0)$ to the equilibrium value $M_{0}$. Adapted from reference [17] with permission.

The $T_{2}$ relaxation time is defined as the time required after excitation for the magnetization to be reduced to $37 \%$ of its initial value via irreversible processes (Fig. 3). This process is described by equation 4.

$$
\mathrm{M}_{\mathrm{X}-\mathrm{Y}}=\mathrm{M}_{0}\left(e^{-\tau / \mathrm{T}_{2}}\right)
$$

where $\tau$ is the amount of time following a radio frequency pulse and $T_{2}$ is the transverse relaxation time. Since the main magnetic field of MRI scanners is not perfectly homogenous, the inhomogeneity of the magnetic field also causes dephasing of individual magnetizations of protons, resulting in more rapid loss of transverse magnetization. The process is called $\mathrm{T}_{2}{ }^{*}$ relaxation.

As the magnetization recovers to the equilibrium, the absorbed energy that is released by these two processes is measured as electrical signals. The position dependent frequency and phase are encoded into the transverse magnetization, and the measured signals are Fourier transformed to construct the spatial image [18]. Relaxation is fundamental to the contrast in an MR image because tissues have different relaxation characteristics. MR images are created based on the proton density, or $\mathrm{T}_{1}$ and $\mathrm{T}_{2}$ relaxation rates, and flow and diffusion properties in different tissues. Tissues with short $T_{1}$ relaxation time give strong MR signal, resulting in bright images in $\mathrm{T}_{1}$-weighted MRI. Tissues with short $T_{2}$ relaxation time produce weak MR signal, resulting in dark images in $\mathrm{T}_{2}$-weighted MRI. In some cases, the difference in relaxation rates between normal and diseased tissues is too small to be detected, resulting in no significant tissue contrast in MR images. Paramagnetic materials, including paramagnetic metal ion chelates and nanomaterials, have been employed as contrast agents to enhance the image contrast by shortening the relaxation times of water protons [19-21].

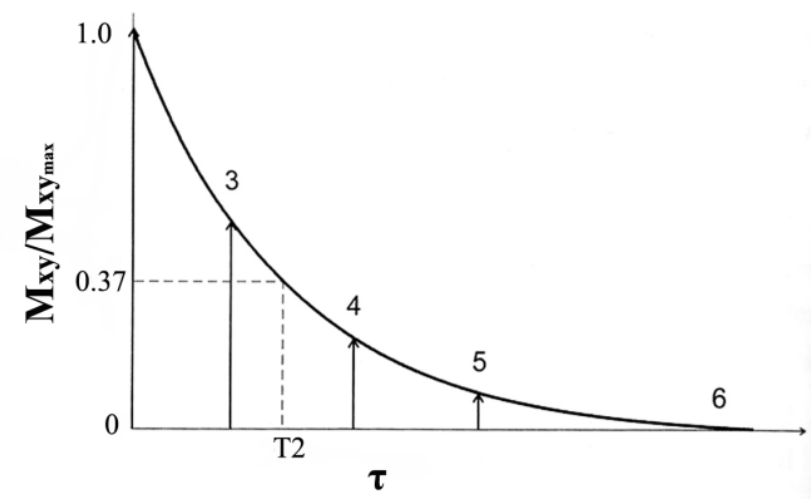

Figure 3. $T_{2}$ relaxation curve. The decay of the magnitude of the transverse magnetization from an initial value. Adapted from reference [17] with permission.

\section{MR contrast agent}

Contrast agents, also known as contrast media, are often used in medical imaging to highlight specific regions (e.g. the blood pool) of the body and make them easier to visualize. In MRI, intensive work in the development of paramagnetic metal contrast agents has been reported in the past few decades [19, 22-24]. There are four main classes of MRI contrast agents based on their different contrast mechanisms, including $\mathrm{T}_{1}$ [19], $\mathrm{T}_{2}$ [25], chemical exchange saturation transfer (CEST) agents [26], and heteronuclear contrast agents [27-29].

$T_{1}$ contrast agents. $T_{1}$ contrast agents shorten the longitudinal relaxation times of surrounding water protons or increase the $T_{1}$ relaxation rate $\left(1 / T_{1}\right)$, and generate a positive image contrast [30]. The increase of $\mathrm{T}_{1}$ relaxation rate is linearly correlated to the concentration of the contrast agents:

$$
1 / \mathrm{T}_{1}=1 / \mathrm{T}_{1, \mathrm{H} 2 \mathrm{O}}+\mathrm{r}_{1}[\mathrm{M}]
$$


where $1 / T_{1}$ is the relaxation rate of water protons with contrast agents, $\mathrm{T}_{1, \mathrm{H} 2 \mathrm{O}}$ is the relaxation time of water protons, $r_{1}$ is the relaxivity of contrast agents, and $[\mathrm{M}]$ is the concentration of contrast agents. A good $T_{1}$ contrast agent is able to shorten $T_{1}$ relaxation times of surrounding water protons even at a low concentration of the agent. This ability is measured by the relaxivity, $r_{1}$, of the contrast agent. Rapid exchange between free water molecules and the water molecules bound to the contrast agents is required for the $T_{1}$ relaxation process and high relaxivity of the contrast agents. A contrast agent with higher $\mathrm{T}_{1}$ relaxivity can help surrounding water protons to recover to the equilibrium longitudinal magnetization more rapidly, producing a brighter image in $\mathrm{T}_{1}$-weighted MRI. Gd(III)-based chelates in Figure 4 are the representative $\mathrm{T}_{1}$-weighted contrast agents.
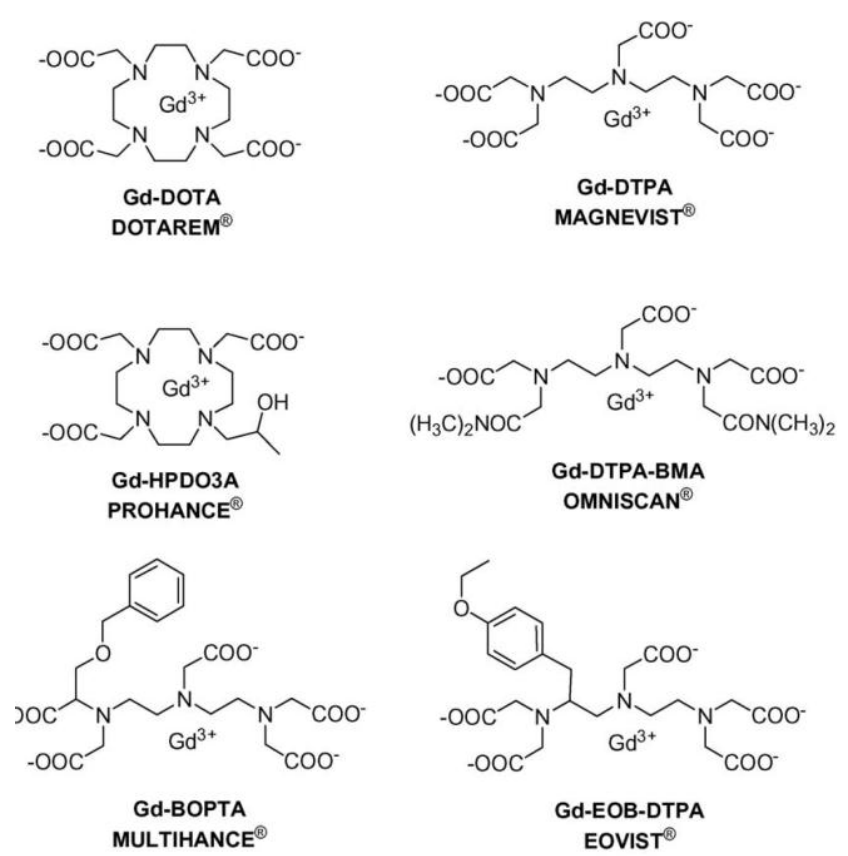

Gd-BOPTA

MULTIHANCE ${ }^{\circledR}$ EOVIST $^{\circledR}$

Figure 4. Schematic illustration of chemical structures of $T_{1}$ contrast agents used in the clinical practice. Adapted from reference [30] with permission.

To date, stable Gd(III) chelates are the commonly used $\mathrm{T}_{1}$-weighted contrast agents in MRI because of their high paramanetism and favorable properties in term of relaxation enhancement [30]. Gd(III) has seven unpaired electrons and their symmetric S-state is closely in tune with the proton's frequency [19]. However, Gd(III) ion is highly toxic after administration as a free ion at low doses $\left(10-20 \mu \mathrm{mol} \mathrm{kg} \mathrm{kg}^{-1}\right)$ [30]. Gd(III) ion needs to be complexed with chelating ligands, e.g. cyclic or linear polyaminocarboxylic acids, forming stable complexes in the design and devel- opment of clinical MRI contrast agents [19]. The first MRI contrast agent approved for clinical use was Gd-(diethylenetriamine pentaacetate) (Gd-DTPA, Magnevist ${ }^{\circledR}$ ). Its clinical applications are initially focused on detecting the breakage of the blood brain barrier in brain tumors [20]. Subsequently, other DTPA derivatives and macrocyclic chelates, including Gd(DTPA-BMA) (Omniscan®), Gd-EOBDTPA (Eovist $\left.{ }^{\circledR}\right)$, Gd-BOPTA (Multihance $\left.{ }^{\circledR}\right)$, Gd-DOTA (Dotarem ${ }^{\circledR}$ ) and Gd(HP-DO3A) (Prohance $\left.{ }^{\circledR}\right)$, have been developed for clinical use, Fig. 4 [31-33]. In addition to $\mathrm{Gd}(\mathrm{III}), \mathrm{Mn}(\mathrm{II})$ has five unpaired electrons and is also paramagnetic. $\mathrm{Mn}(\mathrm{II})$ dipyridoxal diphosphate (Mn-DPDP) was introduced as a hepatocyte-specific contrast agent in clinical practice [34]. These Gd(III) and $\mathrm{Mn}(\mathrm{II})$ complexes, generate brighter images and are the most commonly used $\mathrm{T}_{1}$ MRI contrast media.

$T_{2}$ contrast agents. $T_{2}$ contrast agents shorten $T_{2}$ relaxation times of the surrounding water protons and increase transverse relaxation rates $\left(1 / T_{2}\right)$, resulting in negative image contrast. The enhanced $T_{2}$ relaxation rate of water protons is proportional to the concentration of contrast agents:

$$
1 / \mathrm{T}_{2}=1 / \mathrm{T}_{2, \mathrm{H} 2 \mathrm{O}}+\mathrm{r}_{2}[\mathrm{M}]
$$

where $1 / T_{2}$ is $T_{2}$ relaxation rate with the contrast agents, $T_{2, \mathrm{H}_{2} \mathrm{O}}$ is the relaxation time of water protons, $\mathrm{r}_{2}$ is the relaxivity of contrast agent, and [M] is the concentration of the contrast agents. $T_{2}$ relaxivity, $r_{2}$, measures the ability of contrast agents to affect the $T_{2}$ relaxation rates. $\mathrm{T}_{2}$ contrast agents affect a large number of water molecules by producing strong magnetic susceptibility [35]. Generally, $\mathrm{T}_{2}$ contrast agents have the $r_{2} / r_{1}$ ratios of 10 or more, while $T_{1}$ contrast agents have $r_{2} / r_{1}$ ratios about 1 2 [19].

The most commonly studied $\mathrm{T}_{2}$ contrast agents are superparamagnetic iron oxide (SPIO) particles. Superparamagnetic iron oxide nanoparticles are the first $\mathrm{T}_{2}$ contrast agent developed for clinical applications [36]. In 1996, injectable Feridex ${ }^{\circledR}$ (ferumoxides) was introduced as the world's first organ-specific MR imaging agent for liver imaging. It has been used to detect and evaluate liver lesions associated with an alteration in the reticuloendothelial system [37]. Mono-dispersed superparamagnetic nanoparticles of $\mathrm{Fe}_{3} \mathrm{O}_{4}, \mathrm{CoFe}_{2} \mathrm{O}_{4}$ and $\mathrm{MnFe}_{2} \mathrm{O}_{4}$ with tunable diameters ( 3 to $20 \mathrm{~nm}$ ) have been synthesized through the reaction of metal acetylacetonate and 1,2-hexadecanediol (Fig. 5) [38]. The $\mathrm{MnFe}_{2} \mathrm{O}_{4}$ nanoparticles have higher magnetization and stronger MR contrast effects over $\mathrm{Fe}_{3} \mathrm{O}_{4}$ nanoparticles for in vivo cancer imaging [39-40]. Currently, most of $\mathrm{T}_{2}$ MRI contrast agents are still in the stage of in vitro testing or preclinical animal studies. Several key issues, including toxicological 
effects, rapid excretion after imaging, long-term stability, and pharmacokinetics, have to be addressed prior to further development into clinical applications.

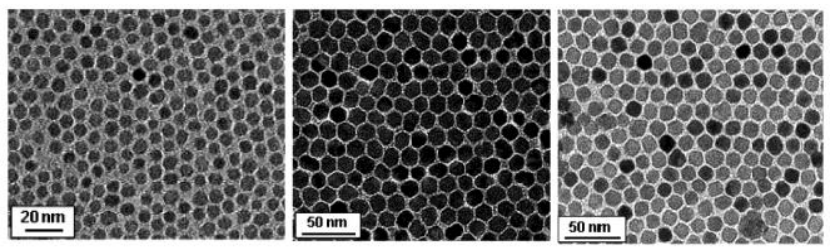

Figure 5. TEM images of $6 \mathrm{~nm} \mathrm{Fe}{ }_{3} \mathrm{O}_{4}$ (left), I $4 \mathrm{~nm} \mathrm{CoFe}{ }_{2} \mathrm{O}_{4}$ (middle) and $14 \mathrm{~nm} \mathrm{MnFe}{ }_{2} \mathrm{O}_{4}$ nanoparticles (right). Adapted from reference [38] with permission.

CEST contrast agents. MR contrast can also be generated by an entirely different mechanism based on chemical exchange saturation transfer [41]. Chemical exchange in NMR refers to any process in which a nucleus exchanges between two or more different chemical environments intramolecularly or intermolecularly [42]. In 1963, Forsén and Hoffman performed the CEST experiment to measure proton transfer rates between salicylaldehyde and water[43]. CEST MRI contrast agents are designed based on the chemical exchange phenomena between bulk water protons $(\delta=4.78 \mathrm{ppm})$ and exchangeable protons with a different chemical shift.

The mechanism of CEST contrast agents is illustrated in Fig. 6. Bulk water protons are considered as pool A, and exchangeable protons of CEST contrast agents are considered as pool $\mathrm{B}$. When the protons in pool B are irradiated with a soft pulse (low-power) at the resonance frequency of a nucleus of interest, the number of protons aligned against the field is increased at the expense of those aligned with the field. Once the number of protons aligned with and against the field is equal, the system is saturated. The net magnetization of pool B is zero and no NMR signal is observed. Due to chemical exchange, a saturated transfer from pool B to pool A takes place, leading to a decrease in signal of bulk water protons in pool A. Longitudinal relaxation returns each system to its equilibrium distribution of protons or steady state. The steady-state intensity $\left(\mathrm{M}_{A \infty} / \mathrm{M}_{A 0}\right)$ of the protons in pool $\mathrm{A}$ is given by equation $7[26,35]$ :

$$
\frac{M_{A \infty}}{M_{A 0}}=\frac{1}{\left(1+k_{2} T_{1 B}\right)}
$$

where $\mathrm{M}_{A \infty}$ is the signal intensity of pool $\mathrm{A}$ after prolonged presaturation of pool $\mathrm{B}$ protons, $\mathrm{M}_{A 0}$ is the initial intensity of pool $\mathrm{A}, k_{2}$ is the unidirectional rate constant for the protons moving from pool A to pool $\mathrm{B}$, and $T_{1 \mathrm{~B}}$ is the longitudinal relaxation time of the protons in pool B. CEST images in MRI are darkened as compared to images obtained without a pre-saturation radio frequency pulse as the result of signal loss in pool A. One significant advantage of CEST agents over typical relaxation agents is that the CEST effect can be switched "on" and "off " at will, since each response is uniquely related to the specific absorption frequency of their mobile protons [44].

A representative CEST spectrum is shown in Fig. 7 (right) using barbituric acid as an example. However, the difference of the chemical shifts of protons in two pools is only a few ppm. In order to increase the sensitivity of CEST MRI, paramagnetic CEST (PATACEST) agents have been developed based on some of the lanthanide chelates [44-47] agents provide larger chemical shifts (Fig. 7, left). The protons of water molecules bound to Eu-DOTA-(glycine ethyl ester) ${ }_{4}$ appear at about 50 ppm away from bulk water protons in the spectrum. Complexes of $\mathrm{Tm}^{3+}$ and $\mathrm{Dy}^{3+}$ are able to shift the bound water peaks to $+500 \mathrm{ppm}$ and -720 ppm, respectively [48]. The lager shift allows for faster exchange rates, resulting in the increase of imaging sensitivity [35]. However, the sensitivity of PARACEST agents is still below that of the conventional Gd(III)-based relaxation agents and the amount of CEST agent required to produce significant water contrast is unrealistically high for clinical applications [49]. Several approaches to enhance the sensitivity of CEST agents have been proposed, including perfluorocarbon-filled nanoparticles [50] entrapped within a liposomal vesicle [30,51], as well as cationic polymer coupled with negatively charged lanthanide complex [52-53].

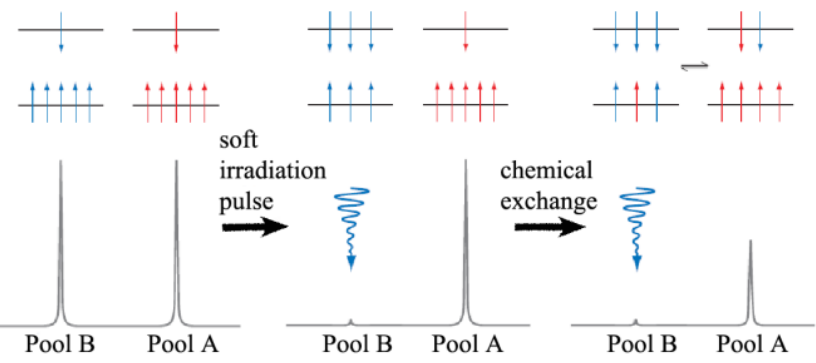

Figure 6. Schematic representations of the distribution of protons, aligned with and against the field (upper and lower energy levels) (top panel) and simulated NMR spectra (lower panel) for two chemically distinct pools of protons (left column), two pools after a saturation pulse has been applied to one pool (middle column), and for a system undergoing chemical exchange after a saturation pulse has been applied to one pool (right column). Adapted from reference [26] with permission. 


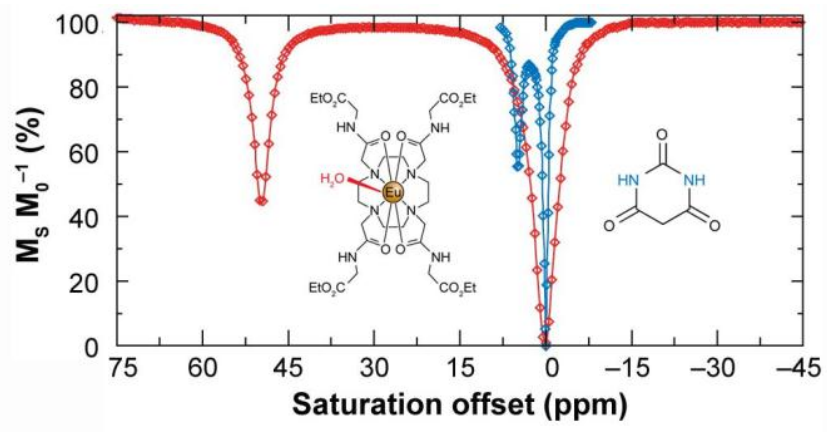

Figure 7. The CEST spectra of barbituric acid (blue) and a $\mathrm{Eu}^{3+}$ complex, Eu-DOTA-(glycine ethyl ester) ${ }_{4}$ (red). The CEST spectrum of barbituric acid is shown on the same scale as that of the $\mathrm{Eu}^{3+}$ complex for comparative purposes. Adapted from reference [26] with permission.

Heteronuclear MRI contrast agents. Proton-based MRI often suffers from interference or low contrast due to the background signals [27]. Relatively high concentration is required for contrast agents to generate significant relaxation enhancement [54] or saturation transfer [55] detectable for MRI[28]. Recently, non-proton contrast agents based on ${ }^{19} \mathrm{~F}[27$, 56-57], and hyperpolarized ${ }^{129} \mathrm{Xe}[28,58-59]$ and ${ }^{13} \mathrm{C}$ $[29,60]$ have been designed for MRI. These new contrast mechanisms based on the signal from nuclei of ${ }^{19} \mathrm{~F}$ or hyperpolarized ${ }^{129} \mathrm{Xe}$ and ${ }^{13} \mathrm{C}$ have a potential to circumvent the limitations of ${ }^{1} \mathrm{H}$-based MRI in contrast sensitivity. For instance, when ${ }^{19} \mathrm{~F}$-containing contrast agents are introduced into living bodies, only the ${ }^{19} \mathrm{~F}$ signal of the contrast agents can be detected without interference from the background. The hyperpolarized ${ }^{129} \mathrm{Xe}$ and ${ }^{13} \mathrm{C}$ MRI is extremely sensitive to the hyperpolarized probes at low concentrations. However, hyperpolarized contrast agents suffer from short in vivo half-lives of the hyperpolarization of the agents. Nevertheless, new contrast mechanisms based on the heteronuclear contrast agents have a potential to expand the scope of MRI in clinical applications.

\section{Integrin Targeted MRI Contrast Agents}

The currently available clinical contrast agents are tissue nonspecific extracellular contrast agents [61]. These agents are not suitable for target-specific contrast enhancement in both clinical and experimental settings [61-63]. Therefore, there have been continuous efforts for developing more specific contrast agents that can offer more accuracy diagnostic imaging [20, 23-24, 61, 64]. Integrins have been used as the molecular targets for design and development of targeted MRI contrast agents.

Integrins are an important family of heterodimeric transmembrane glycoproteins that function as mediators of cell-cell and cell-extracellular matrix interactions. These heterodimeric membrane proteins are highly versatile adhesion receptors, consisting of a large chain $(\alpha)$ of about 1000 amino acids and a small chain $(\beta)$ of about 750 amino acids [65]. A variety of a and $\beta$ subunits assemble into at least 24 distinct integrins[3]. Integrins on the cell surface bind to certain proteins of extracellular matrix, including fibronectin, vitronectin, fibrinogen, collagen, and laminin. The structure of $a$ and $\beta$ subunits of the integrin determines which protein in the extracellular matrix to bind. However, the ligand-binding specificity of the integrins is promiscuous. One integrin can bind several different ligands and several different integrins can bind to the same ligand [65]. For exmple, $a_{v} \beta_{3}$ integrin is the most promiscuous of all known integrins which binds to vitronectin, fibrinogen and fibronectin, whereas $\alpha_{5} \beta_{1}$ and $\alpha_{v} \beta_{3}$ integrins both bind to fibronectin. Integrins are expressed at a cell-type and stage specific manner. Examples of cell-type specific integrins are $\alpha_{\mathrm{II}} \beta_{3}$ in platelets and $\alpha_{6} \beta_{4}$ in epithelial cells. They recognize specific ligands through short amino acid sequences, such as RGD peptide (Arg-Gly-Asp) [66]. Some of the integrins are overexpressed in diseased tissues. For example, overexpression of $\alpha_{v} \beta_{3}$ integrin promotes angiogenesis of in tumor proliferation. The overexpressed integrins can be used as the molecular targets of designing suitable small molecular antagonists as therapeutics and diagnostics for the related diseases [4].

\section{Design of targeted MR contrast agents for mo- lecular imaging of integrins}

A targeted contrast agent includes a targeting agent specific to a target integrin, a paramagnetic agent and a linker connecting the targeting agent with the paramagnetic agent. Proper selection of targeting agents is critical for targeted MRI contrast agents to effectively bind to integrins for in vivo imaging. The commonly used targeting agents are monoclonal antibodies, their fragments and small peptides. Monoclonal antibodies have high specificity for binding to a specific target. Genetically engineered and humanized antibodies have been developed to minimize immune reactions in clinical applications [67-68]. The molecular weight of monoclonal antibodies is approximately $150 \mathrm{KDa}$ and they have a slow blood clearance. Therefore, small size antibody fragments, such as Fab (molecular weight $\sim 50 \mathrm{KDa}$ ) and single-chain variable fragment ( $\mathrm{scFv}$, molecular weight $50 \mathrm{KDa}$ ), have been developed to facilitate penetration into target tissues and to improve targeting efficiency. Synthetic small peptides can also be used as targeting agents in targeted MRI contrast agents. They are less 
immunogenic and can be readily synthesized. RGD peptide is one of commonly used targeting agents for design of targeted imagin agents for $\alpha_{v} \beta_{3}$-integrin [69-72].

Stable Gd(III) chelates can be directly conjugated to the targeting agents in the design of targeted contrast agents. However, due to the low sensitivity of MRI and low concentration of the integrins in target tissues, such targeted contrast agents are not effective for imaging of intergins with MRI. Various carriers, including polymers, dendrimer, liposomes and micelles, have been used in the design of targeted contrast agents to deliver a sufficient amount of Gd(III) chelates at the targeted to generate detectable contrast enhancement by MRI. The Gd(III) chelates must remain chelated in the body and there should be no detectable dissociation of the chelates. Dissociation of the chelates is undesirable because both the free metal and free ligands are generally more toxic than the complex. Superparamagnetic iron oxide nanoparticles can also be incorporated with targeting agents in the design of targeted contrast agents for integrins. Iron oxide nanoparticles often generate strong magnetic susceptibility and are relatively sensitive for $\mathrm{T}_{2}$-weighted MR molecular imaging. After their role of molecular imaging is fulfilled, the targeted agents should be excreted completely from the body within hours to minimize toxicity associated with the contrast agents.

In some cases, steric hindrance due to the close proximity of the targeting agents to the contrast agents may inhibit effective binding of the targeting groups to the target sites. A linker is often used to circumvent this problem in designing targeted MR contrast agents. In addition, a suitable linker can also improve the aqueous solubility and relaxivities of the targeted MR contrast agents. Poly(ethylene glycol) (PEG) is commonly used as a linker because of its insert biophysical properties and good biocompatibility [73]. Avidin-biotin system has a high binding affinity and is also used as a linker in designing targeted MR contrast agents [28, 74-75].

Since blood circulation is turbulent, a targeted contrast agent may have a short time period to encounter a target integrin and the binding between the targeted contrast agent and the integrin may not be strong enough to stop the motion of contrast agents, especially for the bigger size targeted agents, such as macromolecules and nanoparticles. As a result, the targeted agent may not be anchored to the target site. In the design of targeted MRI contrast agents to integrins, it is necessary to incorporate enough ligands into the agents to guarantee the adhesive power of the targeted agents [76]. The integrin targeted MR con- trast agents should be inert substance for in vivo imaging. They should not have any pharmacological and immunological activities after intravenously administration. An ideal integrin targeted MR contrast agent should be non-toxic, provide strong contrast enhancement at the target sites and can be completely excreted from the body after MR imaging.

\section{Targeted MR contrast agents based on small molecular Gd(III) complexes}

Initial efforts to develop targeted MRI contrast agents involve direct conjugation of a signalling group onto an antibody or a peptide. Cyclic RGD peptide has a relatively high and specific affinity for $\alpha_{v} \beta_{3}$-integrin over-expressed in nascent endothelial cells during angiogenesis in various tumor types. Targeted contrast agent specific to $\alpha_{v} \beta_{3}$-integrin has been reported by conjugating table Gd-DOTA chelate to a cyclic RGD peptide [77]. The structure of a RGD targeted contrast agent is shown in Fig. 8 [77]. Gd(DOTA-RGD) has a higher longitudinal relaxivity than Gd-DOTA (Dotarem ${ }^{\circledR}$ ) because of slower molecular tumbling after introduction of the RGD peptide. The targeting ability of Gd(DOTA-RGD) for $\mathrm{a}_{\mathrm{v}} \beta_{3}$-integrin was evaluated with the hepatocellular carcinoma in $\mathrm{H}-\mathrm{ras} 12 \mathrm{~V}$ transgenic mice. Specific enhancement in the tumor with high expression of $\mathrm{a}_{\mathrm{v}} \beta_{3}$-integrin has been observed in the tumor model (Fig. 9).

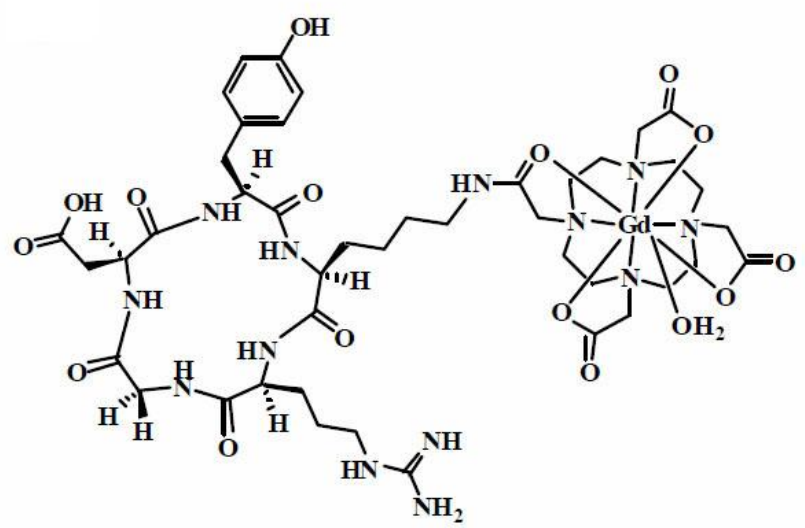

Gd-DOTA-RGD

Figure 8. The chemical structure of Gd-(DOTA-RGD). Adapted from reference [77] with permission. 

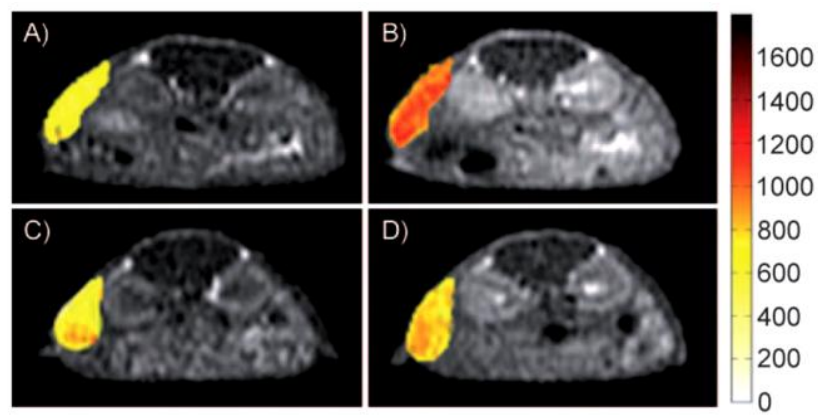

Figure 9. $M R T_{1}$-weighted images of mice with hepatocellular carcinoma before $(A, C)$ and after injection of Gd(DOTA-RGD), (B) and c-(RGDyK)(D) 30 minutes prior the injection of Gd(DOTA-RGD). The color indicates the signal intensity according to the pseudocolor scale on the right. Adapted from reference [77] with permission.

\section{Targeted MR contrast agents based on macro- molecular Gd(III) complexes}

Conjugation of Gd(III) chelates to macromolecules increases the rotational correlation time of a contrast agent, resulting in increased relaxivity [19, 78]. Biocompatible macromolecules have been used as the carriers to conjugate a large number of $\mathrm{Gd}$ (III) chelates and multiple targeting moieties in the development of integrin-specific MRI contrast agents. The targeted macromolecular agents are able to improve MRI sensitivity at the relatively low concentrations of integrins in the targeted sites due to increased relaxivity and a large payload of $\mathrm{Gd}(\mathrm{III})$ chelates.

An $\quad \mathrm{N}$-(2-hydroxypropyl)methacrylamide (HPMA) copolymer-(Gd-DOTA)-cyclic RGD conjugate was prepared for noninvasive $\mathrm{MR}$ imaging of $a_{\mathrm{v}} \beta_{3}$ integrin of MDA-MB-231 breast tumors with quantitative $\mathrm{MR} \mathrm{T}_{1}$-mapping [79]. HPMA copolymers are water soluble, biocompatible and non-immunogenic, and have been used as carriers for drug delivery [80-84]. The structure of the HPMA copolymers targeted MR contrast agent is shown in Fig. 10. The targeted contrast agent was synthesized by free radical copolymerization of HPMA and monomers containing the RGD peptide and Gd chelates using $\mathrm{N}, \mathrm{N}^{\prime}$ - azobisisobutyronitrile as the initiator and 3-mercaptopropionic acid as a chain transfer agent. The $\mathrm{T}_{1}$ relaxivity of HPMA polymer (Gd-DOTA)-RGD conjugate with a molecular weight of $43 \mathrm{KDa}$ was $20.6 \mathrm{~s}^{-1} \mathrm{mM}^{-1}$ per Gd at $1.5 \mathrm{~T}$. The RGD targeted polymer contrast agents resulted in more significant reduction of $T_{1}$ value in tumor tissue 2 hours after the injection in mice bearing MDA-MB-231 tumors than the non-targeted contrast agent, Fig. 11.
The injection of RGD- HPMA copolymer conjugate before that of the targeted agent blocked the binding of the targeted contrast agent to $\alpha_{v} \beta_{3}$-integrin (Fig. 11c).
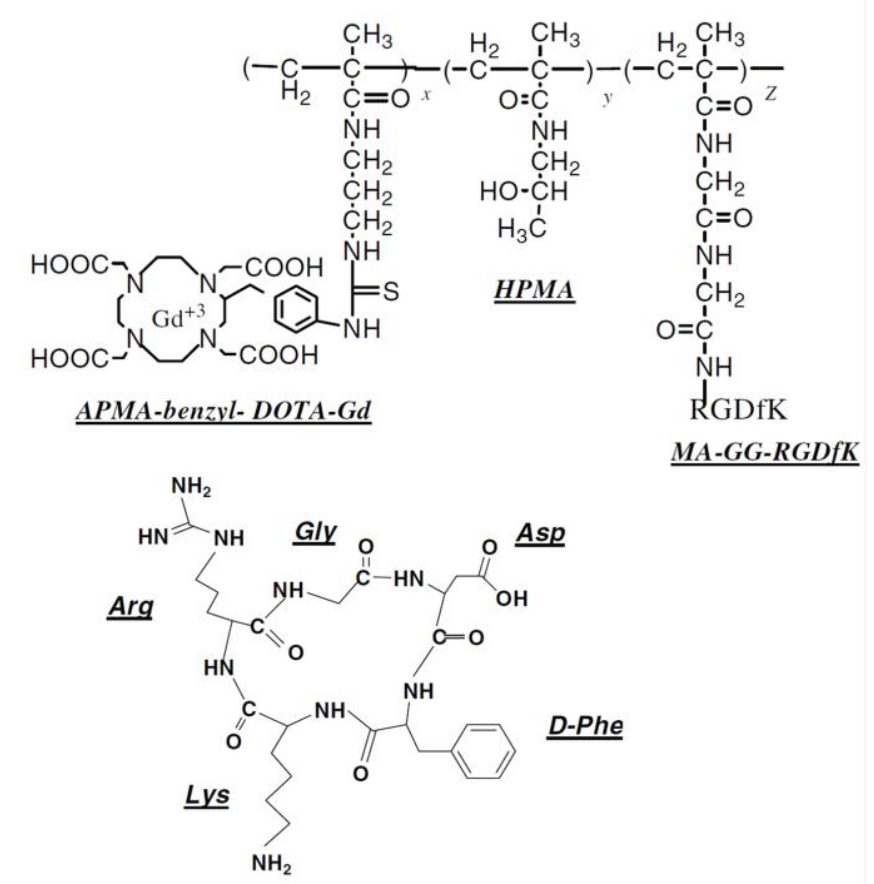

$\underline{R G D f K}$

Figure 10. Structure of HPMA copolymer-(Gd-DOTA)-RGDfK conjugates. HPMA: $\mathrm{N}$-(2-hydroxypropyl)methacrylamide; APMA-benzylDOTA: aminopropyl-methacryl-amide-benzyl-DOTA; MA-GG-RGDfK N-methacryloylglycylglycyl-RGDfK.[77]

A cyclic RGDfK targeted poly $(L$-glutamic acid)-cystamine-(Gd-DO3A) conjugate was reported to facilitate excretion of $\mathrm{Gd}(\mathrm{III})$ chelates after specific imaging of $a_{v} \beta_{3}$-integrin (Fig. 12) [85]. Cystamine was used as a degradable spacer to allow gradual release of $\mathrm{Gd}(\mathrm{III})$ chelates from the conjugate via disulfide-thiol exchange reaction by endogenous free thiols (e.g. cysteine and reduced glutathione) in plasma. The targeted contrast agent exhibited high binding affinity to human prostate carcinoma DU145 cell line. In vivo contrast enhanced MR imaging in tumor bearing mice showed that the RGD targeted polymeric agent resulted in more significant $T_{1}$ reduction at tumor periphery in DU145 prostate cancer xenografts than in sarcoma SLK xenografts due to specific binding to the $\mathrm{a}_{\mathrm{v}} \beta_{3}$-integrin.

Dendrimers are highly branched macromolecules with well-defined structures and sizes. RGD peptide and $\mathrm{Gd}(\mathrm{III})$ chelates have been conjugated to 
polyamidoamine (PAMAM) to prepare targeted contrast agents targeting $\alpha_{v} \beta_{3}$ integrin in angiogenic vessels [86]. An RGD targeted PAMAM conjugate containing both Gd-DTPA and a fluorescent dye was reported for both contrast enhanced MRI and fluorescence imaging [87]. In vitro fluorescence imaging demonstrated the binding of the agent to its molecular target. However, the in vivo biodistribution study using 111In-labeled RGD targeted PAMAM in tumor-bearing mice showed little tumor accumulation. In vivo MR molecular imaging was not pursued with the targeted contrast agent because MRI is far less sensitive than radionuclide detection [87].
A) Pre

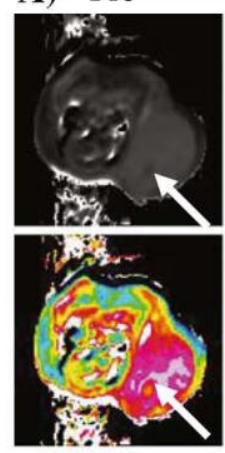

B) Pre

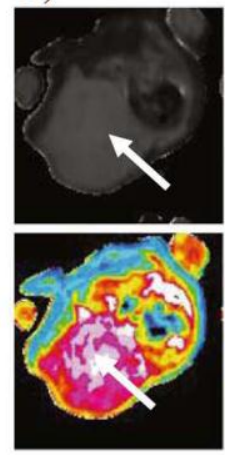

C) Pre

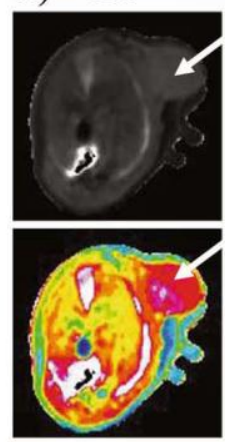

$10 \mathrm{~min}$

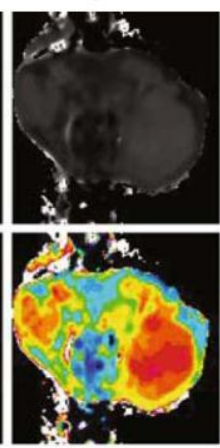

$10 \mathrm{~min}$

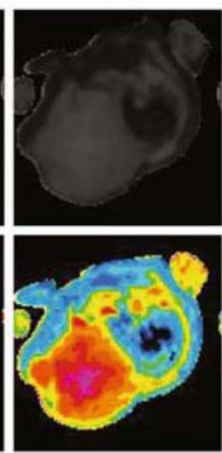

$10 \mathrm{~min}$

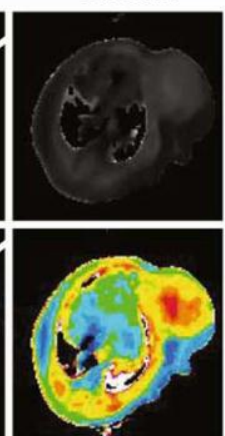

$30 \mathrm{~min}$
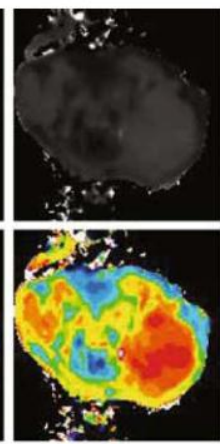

$30 \mathrm{~min}$

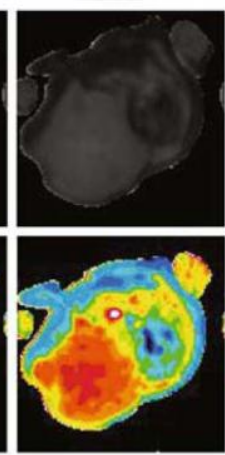

$30 \mathrm{~min}$

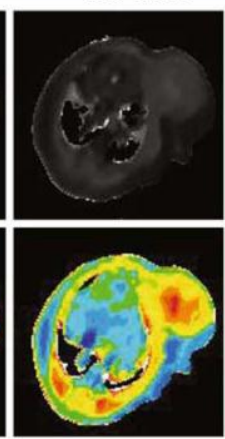

$1 \mathrm{~h}$
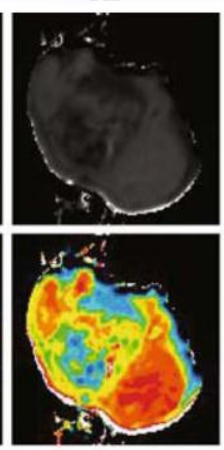

$1 \mathrm{~h}$



$1 \mathrm{~h}$



$2 \mathrm{~h}$
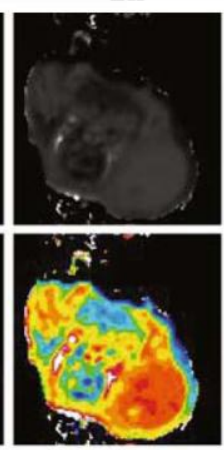

$2 \mathrm{~h}$

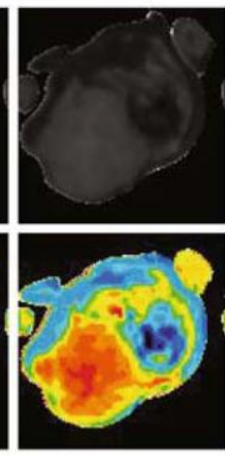

D)
$6 \mathrm{~h}$
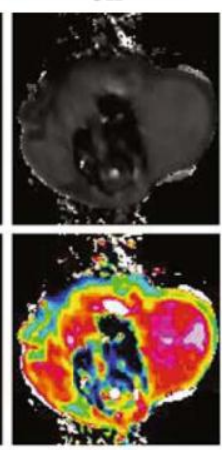

$6 \mathrm{~h}$
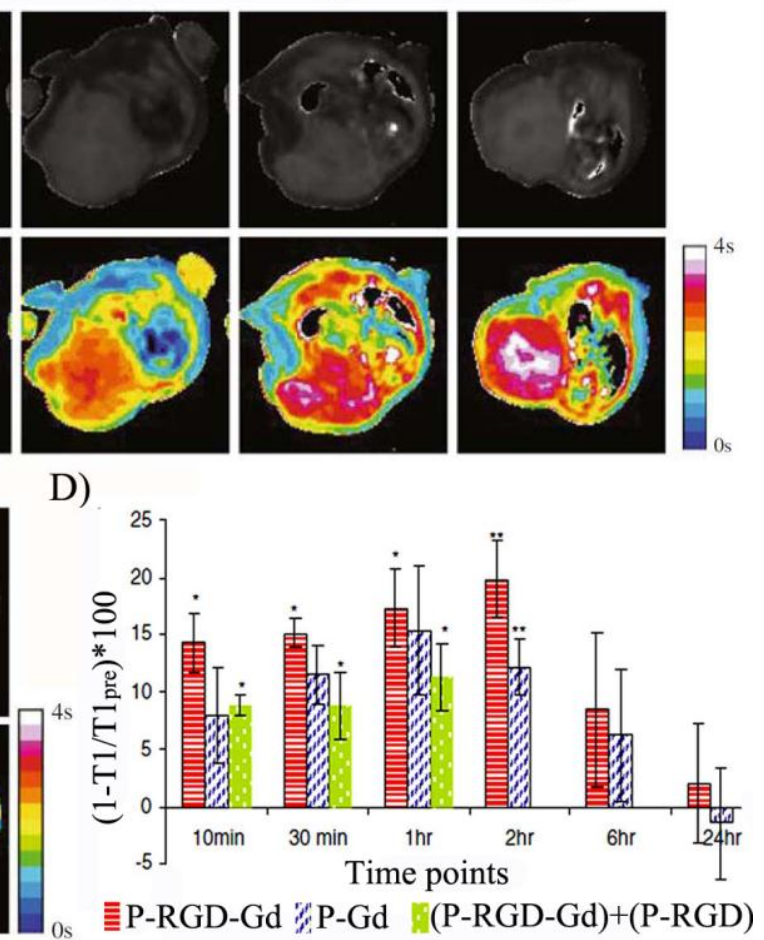

$24 \mathrm{~h}$

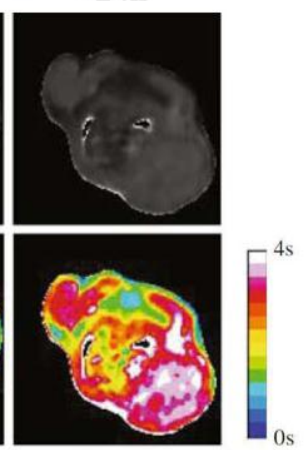

$24 \mathrm{~h}$



$0 \mathrm{~s}$

Figure II. Contrast enhanced axial MR images and $T_{1}$-maps of mice bearing human breast cancer cell line (MDA-MB-23I) xenografts before and after injection of HPMA copolymer-(Gd-DOTA) conjugate (A), HPMA copolymer-(Gd-DOTA)-RGDfK conjugate (B), and HPMA copolymer-(DOTA)-RGDfK conjugate followed by injection of HPMA

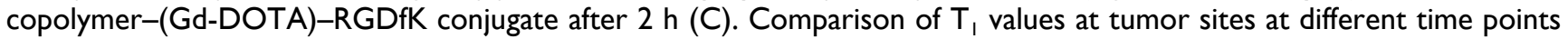
(D). The polymers were injected intravenously at a dose of $0.03 \mathrm{mmol}-\mathrm{Gd} / \mathrm{kg}$. The arrows point to the tumor. The color scales are reflective of $T_{1}$-values. The arrival of the contrast reduces the $T_{1}$-value of the tumor as seen by the color differences and begins to normalize back to its original value after about $6 \mathrm{~h}$. Adapted from reference [77] with permission. 

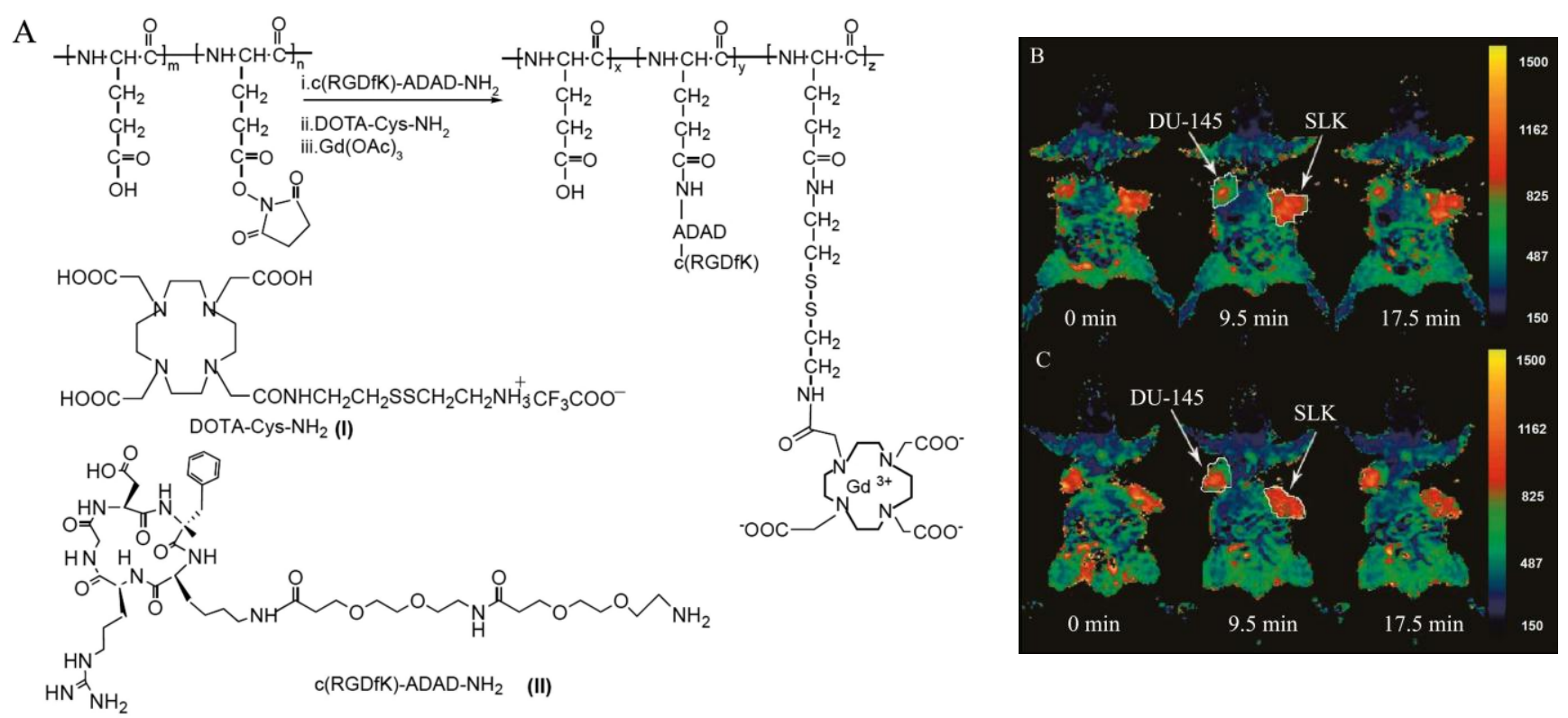

Figure 12. Synthetic scheme of biodegradable $c(R G D f K)$ targeted poly(L-glutamic acid)-cystamine-(Gd-DO3A) conjugate (A). $\mathrm{T}_{1}$-maps of mice bearing human prostate carcinoma DUI45 (left flank) and Kaposi's sarcoma SLK (right flank) xenografts before and after injection of the $c($ RGDfK) containing PGA-cystamine-(Gd-DO3A) conjugate (B) and PGA-cystamine-(Gd-DO3A) (C). Adapted from reference [77] with permission.

\section{Targeted MR contrast agents based on lipid na- noparticles}

Liposomes are spherical and self-assembled bilayers formed by one or more lipids with an inner aqueous phase [88]. Liposomes have been used as a platform for drug delivery and molecular imaging. Paramagnetic chelates or salts, including Gd-DTPA chelates [89]. $\mathrm{MnCl}_{2}$ [90], Gd(DTPA-BMA) [91] and Gd-DO3A [92], have been encapsulated inside the liposomes to prepare MRI contrast agents. Integrin targeting moieties have been coupled to the surface of liposomal MRI contrast agents for contrast enhanced MR imaging of $a_{\mathrm{v}} \beta_{3}$ integrin expressed on cell surface [93]. An $a_{v} \beta_{3}$ integrin specific monoclonal antibody was conjugated to paramagnetic liposomes as a targeted contrast agent for MR imaging of tumor angiogenesis [94]. The paramagnetic polymerized liposomes, shown as the insert of Fig. 13, were conjugated with a monoclonal antibody specific to $\alpha_{v} \beta_{3}$ integrin (LM609) via the biotin-avidin linker. The LM609 antibody-conjugated paramagnetic liposomes (ACPLs) with a size of $300-350 \mathrm{~nm}$ contained $30 \%$ of $\mathrm{Gd}(\mathrm{III})$ chelate-labeled lipid. Tumor contrast enhancement was clearly observed in a rabbit model of squamous cell carcinoma $(\mathrm{V} \times 2)$ with the targeted liposomes, while no significant enhancement was observed in the tumor with the non-specific liposomal contrast agents. The immunohistochemical staining for $\alpha_{v} \beta_{3}$ integrin in the tumor margin was correlated to its adjacent section stained for ACPLs (Fig. 14a, b). The $\alpha_{v} \beta_{3}$-positive vessel density was also correlated well to the degree of MR signal intensity. Strong contrast enhancement was observed in the MR images of tumor tissues with the high density of $a_{v} \beta_{3}$-positive vessels (Fig. 14c, d). RGD peptide was also conjugated to paramagnetic liposomes to develop targeted MRI contrast agent for specific imaging of the tumor angiogenic endothelium in cancer mouse model [95].

An RGD targeted micellar dual imaging agent containing Gd-DTPA and a quantum dot (QD) has been reported for molecular imaging of tumor angiogenesis based on $\alpha_{\mathrm{v}} \beta_{3}$-integrin with optical imaging and MRI [96]. The RGD-targeted micelles were composed of paramagnetic Gd-DTPA-bis(stearylamide) on the micellar surface and CdSe/ZnS core/shell quantum dots in the hydrophobic core (Fig. 15). Significant contrast enhancement was observed at the tumor periphery $45 \mathrm{~min}$ after injection of the RGD-targeted micellar agent in MR images of a mouse tumor model. The strong fluorescence signal in the tumor of fluorescence images (Fig. 15J) further confirmed that the RGD-targeted micelles accumulated in the tumor. 


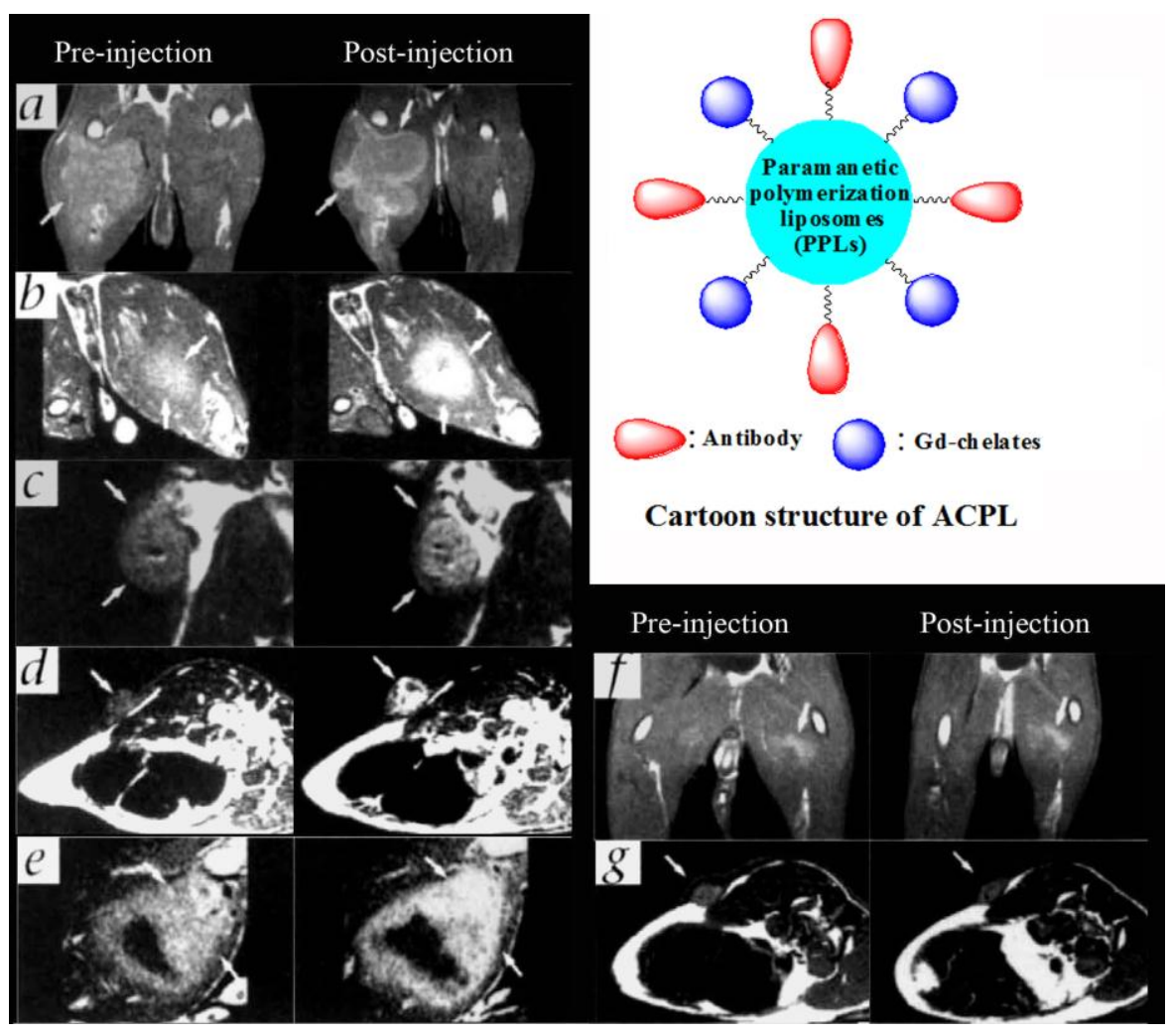

Figure 13. $T, M R$ images of rabbit tumors before and 24 after injection of anti- $\alpha_{v} \beta_{3}$ (LM609) ACPLs. a) Coronal images of tumor in the right thigh muscle; b) axial images of a intramuscle tumor; c) coronal images of a subcutaneously implanted V2 carcinoma; d) LM609 ACPLs improved visualization of a subcutaneous tumor; e) axial images of hyperintense intramuscle tumor with central necrosis and the post-contrast image with LM609 ACPLs; f) coronal image of tumor growing in left thigh muscle and minimal tumor enhancement at $24 \mathrm{~h}$ after injection of isotype-matched control ACPLs; g) axial images of a rabbit subcutaneous tumor and almost no enhancement $24 \mathrm{~h}$ after administration of avidin-conjugated control paramagnetic liposomes. Insert illustrates the structure of ACPL. Adapted from reference [94] with permission.
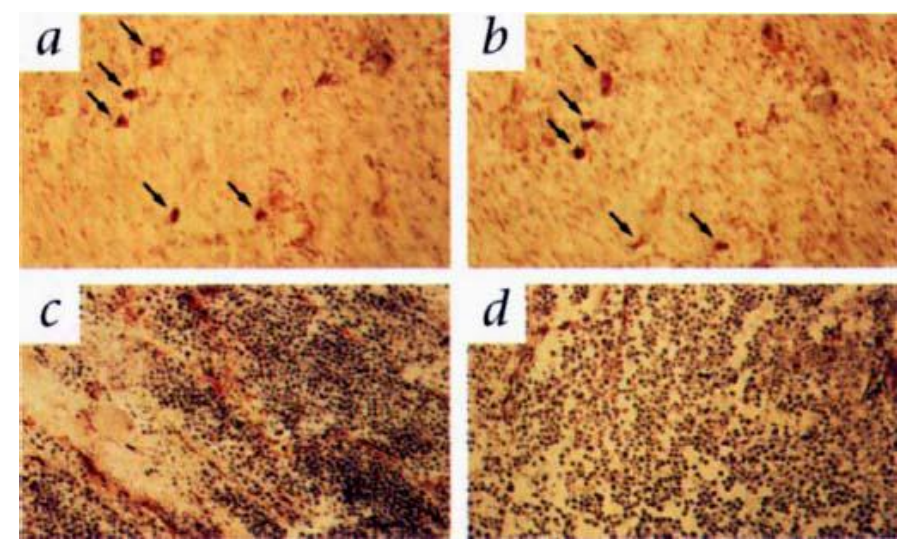

Figure 14. The expression $\alpha_{v} \beta_{3}$ integrin and ACPL localization in tumor tissue sections. a) An immunohistochemical staining section for $\alpha_{v} \beta_{3}$ integrin from the tumor margin shown in Fig. I 3a; b) An adjacent tissue section stained for ACPLs; c) A staining section showed a high density of stained $\alpha_{v} \beta_{3}$ positive vessels from the tumor with strong MR enhancement with the ACPLs; d) A staining section showing a relative paucity of $\alpha_{v} \beta_{3}$ positive vessels from the tumor with relatively weak MR enhancement with ACPLs. Adapted from reference [94] with permission. 

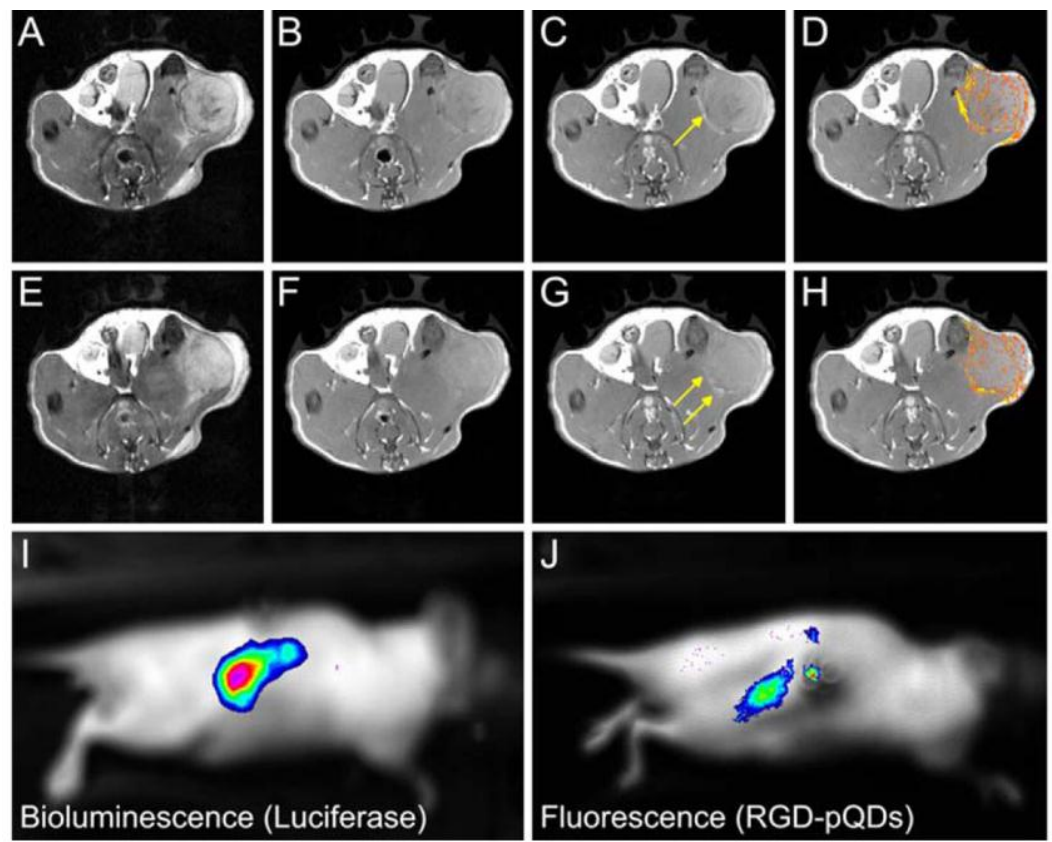

Figure 15. The schematic structure of $\alpha_{v} \beta_{3}$-integrin targeted micellar nanoparticles for optical and MR imaging (left) and $M R$ and optical molecular imaging of tumor angiogenesis (right). $T_{2}$-weighted images before the contrast agent was injected (a, e); $T_{1}$-weighted images before (b, f) and $45 \mathrm{~min}$ after (c, g) the injection of the RGD targeted micellar imaging agent and color-coded signal enhancement in tumors $(d, h)$. The arrows in $(c, g)$ indicate bright regions in the periphery of the tumor. Bioluminescence image (i) and fluorescence image (j) of a nude mouse with a luciferase-expressing renal carcinoma tumor after injection of luciferin (i) and the targeted imaging agent (j). The signal colocalizes with a strong fluorescence signal (j) originating from intravenously administrated RGD-PQDs that are accumulated in the tumor. Adapted from reference [96] with permission.

\section{Targeted MR contrast agents based on super- paramagnetic nanoparticles}

Targeted MRI contrast agents based on superparamagnetic nanoparticles have been reported for $\mathrm{T}_{2}$-weighted $\mathrm{MR}$ imaging of integrins. A targeted $\mathrm{T}_{2}$ MRI contrast agent, c(RGDyK)-4-methylcatechol labeled monodispersed $\mathrm{Fe}_{3} \mathrm{O}_{4}$ nanoparticles, with a diameter of approximately $8.4 \mathrm{~nm}$ was synthesized by thermal decomposition of $\mathrm{Fe}(\mathrm{CO})_{5}$, followed by air oxidation and conjugation with peptide [97]. The $r_{2}$ relaxivity of the $\mathrm{c}(\mathrm{RGDyK})$ targeted $\mathrm{Fe}_{3} \mathrm{O}_{4}$ nanoparticles was $165 \mathrm{mM}^{-1} \mathrm{~s}^{-1}, 60 \%$ increase over that of the commercial Feridex ${ }^{\circledR}$ nanoparticles $\left(104 \mathrm{mM}^{-1} \mathrm{~s}^{-1}\right)$ with a similar core size. The integrin targeting specificity of $\mathrm{c}(\mathrm{RGDyK})$ targeted $\mathrm{Fe}_{3} \mathrm{O}_{4}$ nanoparticles was shown in U87MG human glioblastoma cell line with high $\alpha_{v} \beta_{3}$ integrin expression in comparison with MCF-7 human breast cancer cell line with low $a_{v} \beta_{3}$ integrin expression. The results demonstrated that $\mathrm{Fe}$ uptake in U87MG cells was 5-fold higher than that in MCF-7 cells. A significant MR signal decrease $(\sim 42 \%)$ was observed in $\mathrm{T}_{2}$-weighted MR images of the tumor in mice bearing U87MG xenografts after injection of the $\mathrm{c}(\mathrm{RGDyK})$ targeted $\mathrm{Fe}_{3} \mathrm{O}_{4}$ nanoparticles (Fig. 16A, B). The competitive study in the presence of free c(RGDyK) peptide only resulted in less signal reduction $(\sim 15 \%)$ for the targeted agent (Fig. 16C). Prussian blue staining confirmed the accumulation of the targeted $\mathrm{Fe}_{3} \mathrm{O}_{4}$ nanoparticles in the tumor tissue after the MR scan at $4 \mathrm{~h}$ post-injection (Fig.16 D). The presence of the free peptide significantly reduced Prussian blue staining in tumor tissue (Fig. 16 E). 

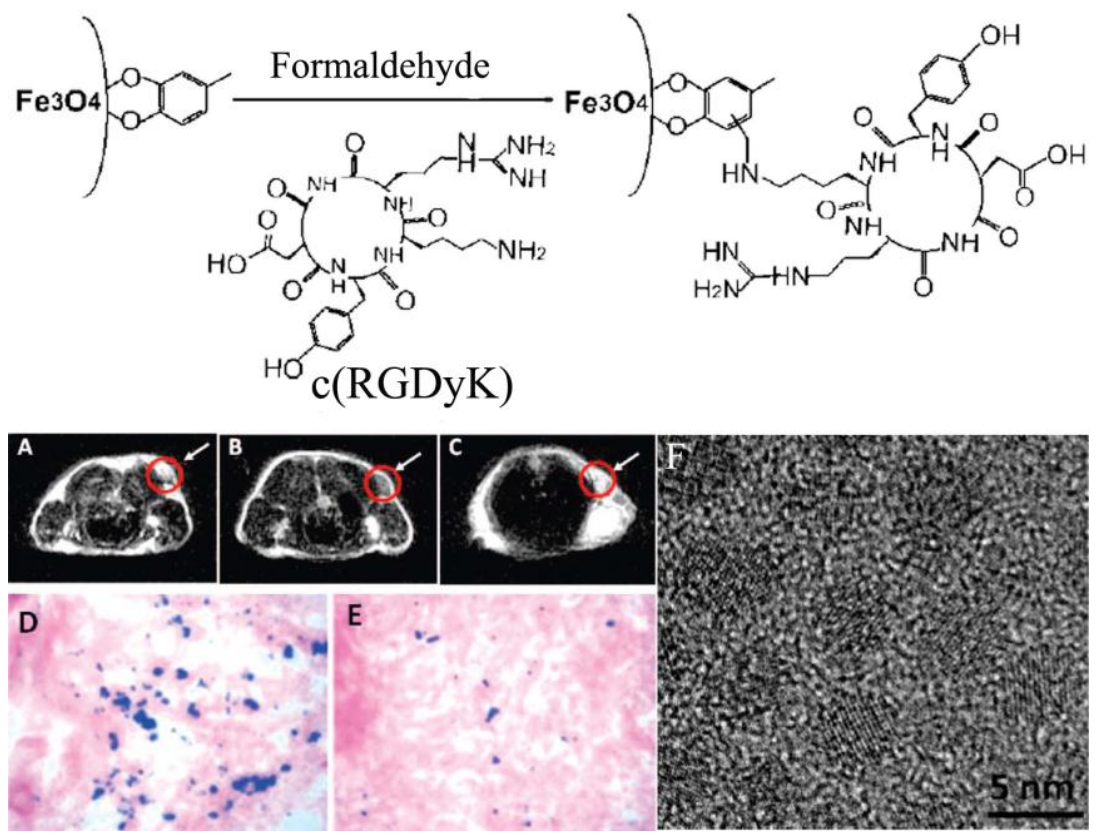

Figure 16. Schematic illustration of coupling $c(R G D y K)$ peptide to $\mathrm{Fe}_{3} \mathrm{O}_{4}$ nanoparticles (upper). $A x i a l \mathrm{~T}_{2}$-weighted $\mathrm{MR}$ images of the U87MG tumors implanted in mice without nanoparticles (A), with injection of $300 \mu \mathrm{g}$ of c (RGDyK)-MC- $-\mathrm{Re}_{3} \mathrm{O}_{4}$ nanoparticles (B) and with the injection of $c(R G D y K)-M C-\mathrm{Fe}_{3} \mathrm{O}_{4}$ nanoparticles free $\mathrm{c}(\mathrm{RGDyK})(\mathrm{C})$. Prussian blue staining of U87MG tumors for $\mathrm{c}(\mathrm{RGDyK})-\mathrm{MC}-\mathrm{Fe}_{3} \mathrm{O}_{4}$ nanoparticles (D), both (RGDyK)-MC- $\mathrm{Fe}_{3} \mathrm{O}_{4}$ nanoparticles and free $\mathrm{c}(\mathrm{RGDyK})$ (E). High resolution TEM image of $4.5 \mathrm{~nm} \mathrm{MC}-\mathrm{Fe}_{3} \mathrm{O}_{4}$ nanoparticles (F). Adapted from reference [97] with permission.

RGD targeted bi-functional imaging probes have also been developed based on iron oxide for molecular imaging of integrins with dual imaging modalities. Cyclic RGD peptide and ${ }^{64} \mathrm{Cu}$-DOTA have been incorporated onto the surface of iron oxide nanoparticles for imaging of $\alpha_{v} \beta_{3}$-integrin with PET and MRI [98]. The targeted bifunctional imaging agent resulted in strong PET signal in U87MG xenografts with overexpressed $\alpha_{v} \beta_{3}$-integrin in a mouse tumor model and significant tumor contrast enhancement in $\mathrm{T}_{2}$-weighted MRI. An RGD targeted bi-functional agent containing iron oxide nanoparticles and a near-infrared fluorescent (NIRF) dye IRDye800 have also been reported for imaging of $\alpha_{v} \beta_{3}$-integrin with fluorescence imaging and MRI [99]. Both RGD and IRDye800 were coupled onto iron oxide nanoparticles via a triblock copolymer coated on their surface (Fig. 17a). Iron oxide nanoparticle core has a diameter of approximately $10 \mathrm{~nm}$ (Fig. 17b). An amphiphilic PEGylated triblock copolymer consisting of a polybutylacrylate segment, a polyethylacrylate segment, a polymethacrylic acid segment and a hydrophobic hydrocarbon side chain was coated onto the iron oxide nanoparticles, forming a bilayered structure and rendering the nanoparticles water-soluble. IRDye 800 possesses improved tissue penetration ability as compared to conventional fluorophores in the visible range. Approximately 10 IRDye 800 molecules and 15 RGD peptides were coupled onto each nanoparticle. The hydrodynamic size of the targeted agent was approximately $20 \mathrm{~nm}$ as determined by dynamic light scattering analysis. The $r_{2}$ relaxivity of the agent was $190 \mathrm{~mm}^{-1} \mathrm{~s}^{-1}$ (Fig. 17c,d). The targeted dual imaging agent resulted in strong fluorescence signal in U87MG tumor tissue in the fluorescence images than the non-targeted contrast agent. Correspondingly, the targeted agent resulted in more significant $T_{2}$ enhancement in the tumor tissue than the non-targeted agent in the $\mathrm{T}_{2}$-weighted images of the tumor bearing mice (Fig. 18). High sensitive PET and fluorescence imaging with the targeted duel imaging agents validate the effectiveness of the targeted iron oxide nanoparticles in $T_{2}$ weighted $M R$ imaging of $\mathrm{a}_{\mathrm{v}} \beta_{3}$-integrin overexpressed in tumor tissue.

Recently, cyclic(RGDfK)-targted superparamagnetic polymeric micells (SPPM, Fig. 19A) have been developed for sensitive molecular imaging of $\mathrm{a}_{\mathrm{v}} \beta_{3}$ integrin in cancer with MRI using off-resonance saturation [100]. Hydrophobic, mono-dispersed SPIO with a diameter $\sim 9.9 \mathrm{~nm}$ were 
encapsulated inside of the micelles, and the peptide was attached onto the micelles' surface. The final diameter of SPPM particles was about $75 \mathrm{~nm}$ determined by TEM (Fig. 19C). The mechanism of off-resonance saturation (ORS) is similar to CEST, and a presuturation pulse can be applied to turn "ON" /"OFF" the contrast agents as desired. The difference of the ORS is that it presaturates at an off-resonance frequency position away from the bulk water, while a specific exchangeable proton frequency is presaturated for CEST. In the absence of SPPM, the presaturation radiofrequency pulse has little effect on the signal intensity of SPPM-free water (Fig. 19B, top). In the presence of SPPM, the RF pulse can saturate a larger volume fraction of water molecules due to proton relaxation by SPPM, leading to a considerable decrease in signal intensity (Fig. 19B, bottom). ORS contrast can be significantly amplified because of the rapid diffusion of water molecules [101]. Significant peak broadening was observed with increasing Fe concentrations, as shown by ${ }^{1} \mathrm{H}$-nuclear MR spectra of aqueous solutions containing different concentrations of SPPM particles in Fig. 19D.

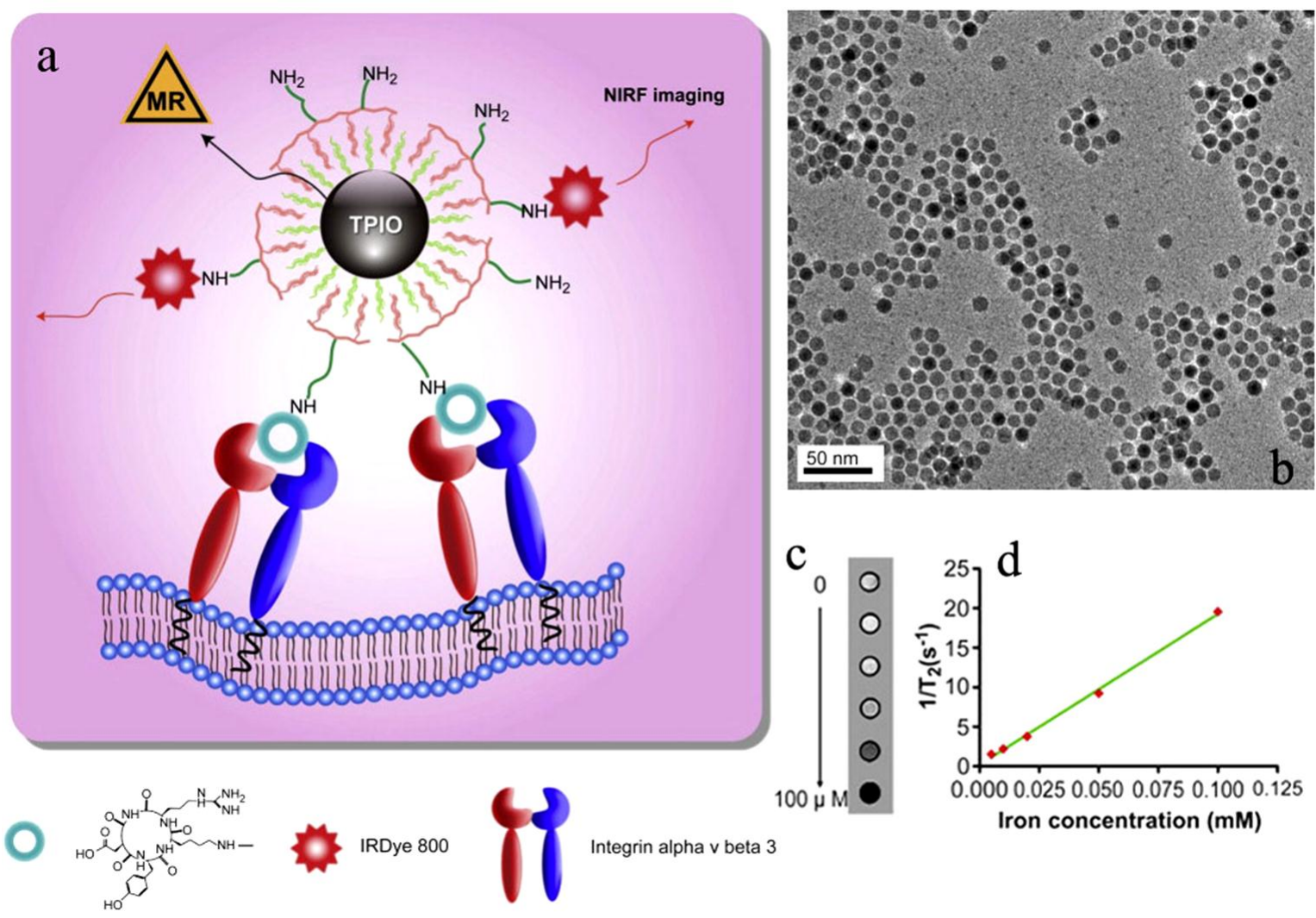

Figure I7. a) Schematic illustration of dual-modality RGD targeted iron oxide nanoparticles containing IRDye800 for tumor $\alpha_{v} \beta_{3}$ integrin imaging. b) TEM of the $10 \mathrm{~nm}$ iron oxide nanoparticles. c) $T_{2}$-weighted phantom images of targeted agent at increased iron concentrations. d) $\mathrm{I} / \mathrm{T}_{2}$ vs. Fe concentration plot of the targeted agent. Adapted from reference [99] with permission. 


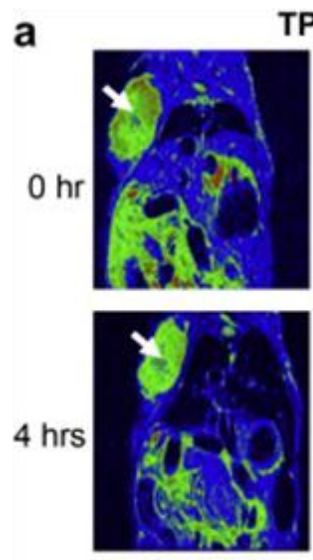

Coronal
TPIO
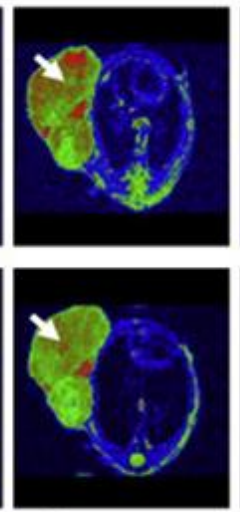

Transverse
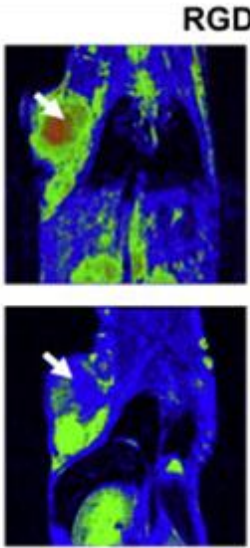

Coronal


Transverse
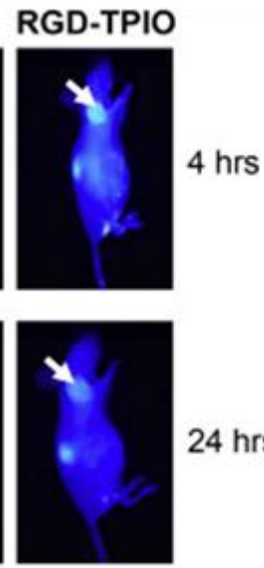

$24 \mathrm{hrs}$

Figure 18. a) MR images of U87MG tumor-bearing mice injected with the RGD targeted dual agent (RGD-TPIO) and the nontargeted agent (TPIO) at a dose of $10 \mathrm{mg} \mathrm{Fe} / \mathrm{kg}$. The images were taken both coronally and transversely before and $4 \mathrm{~h}$ after particle injection. b) Optical imaging of U87MG tumor-bearing mice injected with RGD-TPIO and TPIO. The images were acquired $4 \mathrm{~h}$ hh $\mathrm{h}$ post injection. Adapted from reference [99] with permission.
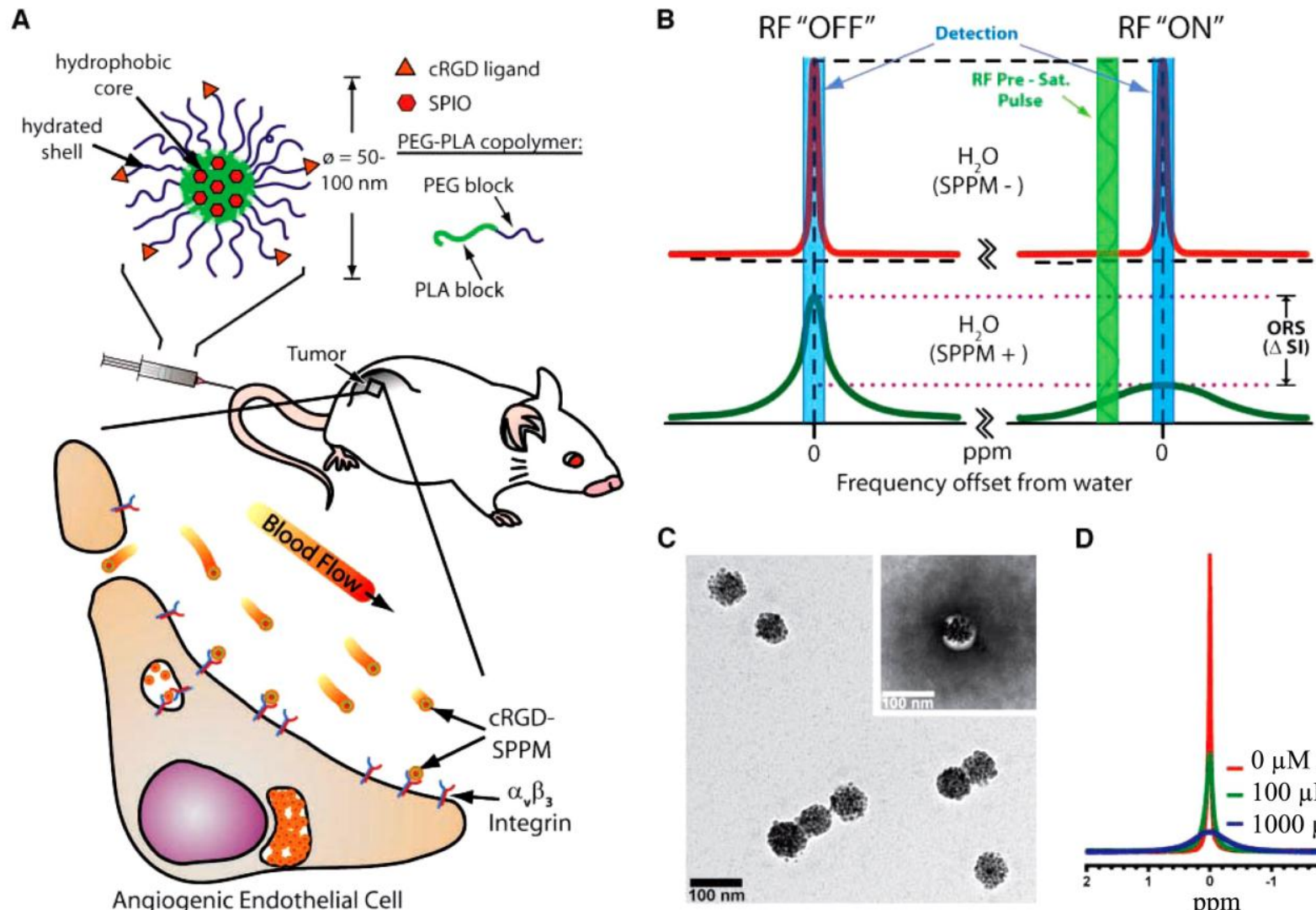

C

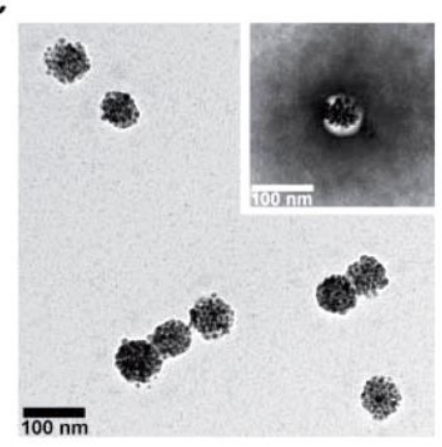

D

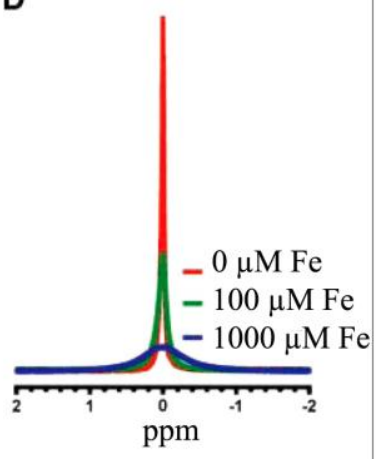

Figure 19. Cancer molecular imaging with cRGD-targeted SPPM and off-resonance saturation (ORS) MRI. A, schematic illustration of a cRGD-encoded SPPM and its targeting to $\alpha_{v} \beta_{3}$-integrin expressing endothelial cells in the tumor vasculature. $B$, mechanism of SPPM-induced ORS contrast. Presaturation RF pulse results in significant decrease in signal intensity in SPPM(+) $\mathrm{H}_{2} \mathrm{O}$ over SPPM(-) $\mathrm{H}_{2} \mathrm{O}$. C, TEM image of a representative SPPM sample. D, IH-NMR $(300 \mathrm{MHz})$ spectra of water containing different concentrations of SPPM (in $[\mathrm{Fe}] / \mu \mathrm{mol} / \mathrm{L}$ ). Adapted from reference [100] with permission. 
Molecular imaging of RGD targeted SPPM with ORS MRI was demonstrated in mice bearing A549 tumor xenografts (Fig. 20). As shown in Fig. 20A, ORS contrast images showed a significant enhancement in tumors by cRGD-targeted SPPM $1 \mathrm{~h}$ after the injection. The CNR of the tumor over background muscle tissue was 10.7, which was larger than that of control groups injected with non-targeted SPPM and a mixture of free RGD with RGD targeted SPPM (Fig. 20B).

Prussian blue staining showed more accumulation of SPPM in tumor vasculature for cRGD-targeted
SPPM than the controls (Fig. 20A). The cRGD-targeted SPPM showed more rapid blood clearance than cRGD-free SPPM injected over 24 hours (Fig. 20C). Significantly higher tumor accumulation was observed for the cRGD-targeted SPPM than the controls 1 hour after administration of the SPPMs (Fig. 20D). ORS MRI with the targeted SPPM showed much higher sensitivity for imaging of $\alpha_{v} \beta_{3}$ integrin in angiogenic tumors than traditional $\mathrm{T}_{2}{ }^{*}$-weighted MRI.
A

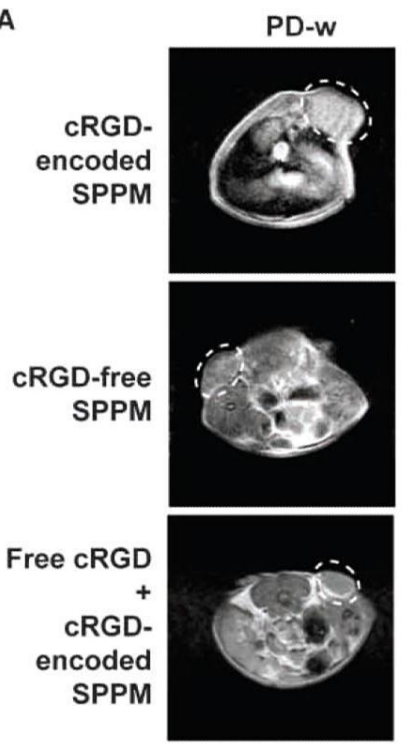

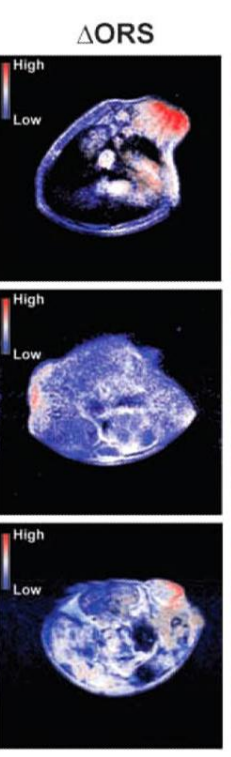

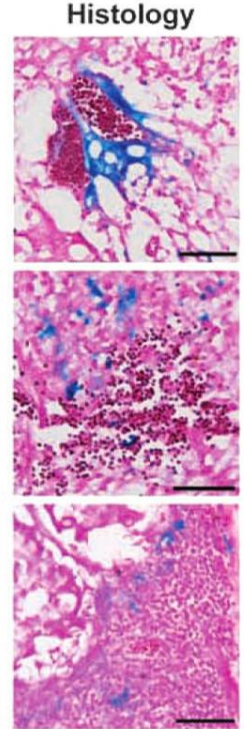

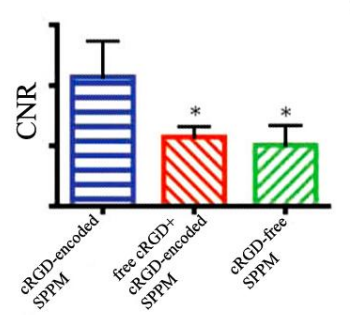



D

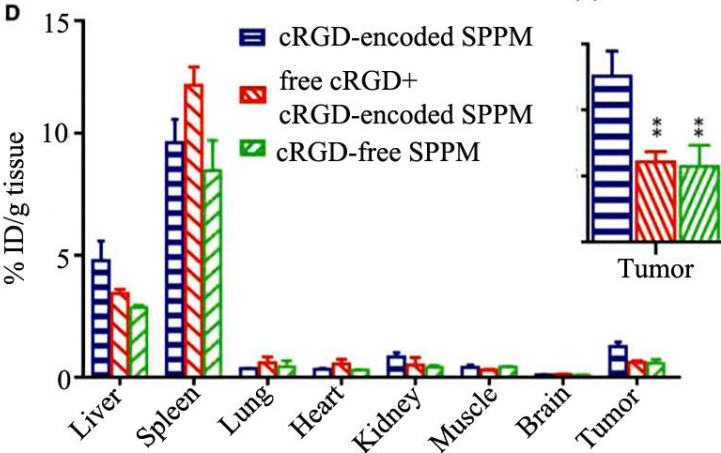

Figure 20. Proton density-weighted $M R$ images (PD-w) and ORS MR images of $\alpha_{v} \beta_{3}$ integrin in tumor tissue with CRGD-targeted SPPM, cRGD-free SPPM, and a mixture of cRGD-encoded SPPM with free CRGD peptide in mice bearing A549 tumor xenografts (6 mg Fe/kg, free peptide 18 molar excess), and Prussian blue staining showing Fe presence from SPPM samples in tumor tissues (A). Comparisons of CNRs of $\triangle$ ORS images of tumor xenograft $(n=4)$ injected with cRGD-encoded SPPM, cRGD-free SPPM, and a mixture of cRGD-encoded SPPM with free cRGD peptide (B). Plasma concentration versus time for CRGD-encoded SPPM and cRGD-free SPPM (C). Biodistribution $(n=3)$ of $c R G D$-encoded SPPM, cRGD-free SPPM, and cRGD-encoded SPPM coinjected with free cRGD I h after i.v. administration. The asterisks (* in $B$ and ** in $D$, inset) indicate statistical significance $(P \leq 0.05)$ between the SPPM group of interest and cRGD-targeted SPPM based on the Student's t test. Adapted from reference [100] with permission.

\section{Conclusion}

MRI is clinical diagnostic imaging modality without ionizing radiation and visualizes soft tissues with high spatial resolution. Contrast enhanced MR imaging has potential to visualize the over-expression of integrins in pathological tissues for accurate diagnosis and evaluation of therapeutic efficacy. Unfor- tunately, MRI in molecular imaging is limited by its relatively low sensitivity. Various targeted contrast agents have been designed and prepared for molecular imaging of integrins with MRI. By using proper delivery systems for loading sufficient Gd(III) chelates or superparamagnetic nanoparticles, effective molecular imaging of integrins with MRI has been demonstrated in animal models. 
Sensitivity of contrast enhanced MRI can be improved using new contrast mechanisms, including off-resonance saturation and hyperpolarization. The combination of MRI with complementary imaging techniques such as fluorescence imaging may provide more effective imaging of integrins. Clinical development of MR imaging of integrins can be achieved after the demonstration of the safety and effectiveness of the integrin-specific contrast agents.

\section{Conflict of Interest}

The authors have declared that no conflict of interest exists.

\section{Acknowledgment}

This work is supported in part by the NIH R01 CA097465 grant.

\section{References}

1. Caravan P. Protein-targeted gadolinium-based magnetic resonance imaging (MRI) contrast agents: design and mechanism of action. Acc Chem Res. 2009;42:851-62.

2. Morawski AM, Lanza GA, Wickline SA. Targeted contrast agents for magnetic resonance imaging and ultrasound. Curr Opin Biotechnol. 2005;16:89-92.

3. Hynes RO. Integrins: bidirectional, allosteric signaling machines. Cell. 2002;110:673-87.

4. Mousa SA. Anti-integrin as novel drug-discovery targets: potential therapeutic and diagnostic implications. Curr Opin Chem Biol. 2002;6:534-41.

5. Jin H, Varner J. Integrins: roles in cancer development and as treatment targets. Brit J Cancer. 2004;90:561-5.

6. Brooks P, Clark R, Cheresh D. Requirement of vascular integrin av $\beta 3$ for angiogenesis. Science. 1994;264:569-71.

7. Niewiarowska J, Sacewicz I, Wiktorska M, Wysocki T, Stasikowska O, Wagrowska-Danilewicz M, et al. DNAzymes to mouse $\beta 1$ integrin mRNA in vivo: targeting the tumor vasculature and retarding cancer growth. Cancer Gene Ther. 2009;16:713-22.

8. Kurokawa A, Nagata M, Kitamura N, Noman A, Ohnishi M, Ohyama $T$, et al. Diagnostic value of integrin $\alpha 3, \beta 4$, and $\beta 5$ gene expression levels for the clinical outcome of tongue squamous cell carcinoma. Cancer. 2008;112:1272-81.

9. Kumar C. Integrin $\operatorname{av} \beta 3$ as a therapeutic target for blocking tumor-induced angiogenesis. Curr Drug Targets. 2003;4:123-31.

10. Lauterbur PC. Image formation by induced local interactions: examples of employing nuclear magnetic resonance. Nature. $1973 ;: 242$

11. Damadian R GM, Minkoff L. NMR in cancer: XVI. Fonar image of the live human body. Physiol Chem Phys. 1977;9:97-100.

12. Young I, Clarke G, Bailes D, Pennock J, Doyle F, Bydder G. Enhancement of relaxation rate with paramagnetic contrast agents in NMR imaging. CT-J Comput Tomogr. 1981;5:543-7.

13. Yuan C, Skinner M, Kaneko E, Mitsumori L, Hayes C, Raines E, et al. Magnetic resonance imaging to study lesions of atherosclerosis in the hyperlipidemic rabbit aorta. Magn Reson Imaging. 1996;14:93-102.

14. Yuan C, Mitsumori L, Beach K, Maravilla K. Carotid atherosclerotic plaque: Noninvasive MR characterization and identification of vulnerable lesions. Radiology. 2001;221:285-99.

15. Yuan C, Kerwin W. MRI of atherosclerosis. J Magn Reson Imaging. 2004;19:710-9.
16. Macdonald G, Peduto A. Magnetic resonance imaging (MRI) and diseases of the liver and biliary tract. Part 1. Basic principles, MRI in the assessment of diffuse and focal hepatic disease. J Gastroen Hepatol. 2000;15:980-91.

17. Brown MA, Semelka RC. MRI basic principles and applications, 3rd ed. Hoboken New Jersey: John Wiley\&Sons, Inc. 2003.

18. Liang ZP. Principles of magnetic resonance imaging: a signal processing perspective. New York: SPIE Optical Engineering Press; 2000.

19. Caravan P, Ellison J, McMurry T, Lauffer R. Gadolinium(III) chelates as MRI contrast agents: Structure, dynamics, and applications. Chem Rev. 1999;99:2293-352.

20. Na H, Song I, Hyeon T. Inorganic Nanoparticles for MRI Contrast Agents. Adv Mater. 2009;21:2133-48.

21. Hermann P, Kotek J, Kubicek V, Lukes I. Gadolinium(III) complexes as MRI contrast agents: ligand design and properties of the complexes. Dalton Trans. 2008;:3027-47.

22. Yurt A, Kazanci N. Investigation of magnetic properties of various complexes prepared as contrast agents for MRI. J Mol Struct. 2008;892:392-7.

23. Werner E, Datta A, Jocher C, Raymond K. High-relaxivity MRI contrast agents: where coordination chemistry meets medical imaging. Angew Chem Int Ed Engl. 2008;47:8568-80.

24. Jacques V, Desreux J. New classes of MRI contrast agents. Top Curr Chem. 2002;221:123-64.

25. Laurent S, Forge D, Port M, Roch A, Robic C, Elst L, et al. Magnetic iron oxide nanoparticles: Synthesis, stabilization, vectorization, physicochemical characterizations, and biological applications. Chem Rev. 2008;108:2064-110.

26. Sherry A, Woods M. Chemical exchange saturation transfer contrast agents for magnetic resonance imaging. Annu Rev Biomed Eng. 2008;10:391-411.

27. Mizukami S, Takikawa R, Sugihara F, Hori Y, Tochio H, Walchli $\mathrm{M}$, et al. Paramagnetic relaxation-based F-19 MRI probe to detect protease activity. J Am Chem Soc. 2008;130:794-5.

28. Schroder L, Lowery T, Hilty C, Wemmer D, Pines A. Molecular imaging using a targeted magnetic resonance hyperpolarized biosensor. Science. 2006;314:446-9.

29. Reineri F, Viale A, Giovenzana G, Santelia D, Dastru W, Gobetto R, et al. New Hyperpolarized Contrast Agents for C-13-MRI from Para-Hydrogenation of Oligooxyethylenic Alkynes. J Am Chem Soc. 2008;130:15047-53.

30. Aime S, Crich S, Gianolio E, Giovenzana G, Tei L, Terreno E. High sensitivity lanthanide(III) based probes for MR-medical imaging. Coord Chem Rev. 2006;250:1562-79.

31. Schmitt-Willich H, Brehm M, Ewers C, Michl G, Muller-Fahrnow A, Petrov $\mathrm{O}$, et al. Synthesis and physicochemical characterization of a new gadolinium chelate: The liver-specific magnetic resonance imaging contrast agent Gd-EOB-DTPA. Inorg Chem. 1999;38:1134-44.

32. Uggeri F, Aime S, Anelli P, Botta M, Brocchetta M, Dehaen C, et al. Novel contrast agents for magnetic-resonance-imaging synthesis and characterization of the ligand BOPTA and its $\ln (\mathrm{III})$ complexes $(\mathrm{ln}=\mathrm{Gd}, \mathrm{La}, \mathrm{Lu})$ - $\mathrm{x}$-ray structure of disodium (tps-9-145337286-c-s)-[4-carboxy-5,8,11-tris(carboxymethyl)-1-p henyl-2-oxa-5,8,11-triazatridecan-13-oato(5-)] gadolinate(2-) in a mixture with its enantiomer. Inorg Chem. 1995;34:633-42.

33. Weinmann HJ, Mühler A, Radüchel B, Young iIR. Biomedical Magnetic Resonance Imaging and Spectroscopy. Chichester: John Wiley \& Sons Ltd.,; 2000.

34. Rummeny E, Marchal G. Liver imaging - Clinical applications and future perspectives. Acta Radiol. 1997;38:626-30.

35. Khemtong C, Kessinger C, Gao J. Polymeric nanomedicine for cancer MR imaging and drug delivery. Chem Comm (Camb). $2009 ;: 3497-510$ 
36. Dias M, Lauterbur P. Ferromagnetic particles as contrast agents for magnetic-resonance-imaging of liver and spleen. Magn Reson Med. 1986;3:328-30.

37. [Internet] Web page. http://imaging.bayerhealthcare.com /html/feridex/index.html.

38. Sun S, Zeng H, Robinson D, Raoux S, Rice P, Wang S, et al. Monodisperse $\mathrm{MFe}_{2} \mathrm{O}_{4}(\mathrm{M}=\mathrm{Fe}, \mathrm{Co}, \mathrm{Mn})$ nanoparticles. J Am Chem Soc. 2004;126:273-9.

39. Lee J, Huh Y, Jun $\mathrm{Y}$, Seo J, Jang J, Song H, et al. Artificially engineered magnetic nanoparticles for ultra-sensitive molecular imaging. Nat Med. 2007;13:95-9.

40. Xu C, Sun S. Superparamagnetic nanoparticles as targeted probes for diagnostic and therapeutic applications. Dalton Trans. 2009;:5583-91.

41. Ward K, Aletras A, Balaban R. A new class of contrast agents for MRI based on proton chemical exchange dependent saturation transfer (CEST). J Magn Reson. 2000;143:79-87.

42. Roberts GCK. NMR of Macromolecules: A Practical Approach (Practical Approach Series) (Hardcover). USA: Oxford University Press; 1993.

43. Forsén S, Hoffman R. Study of moderately rapid chemical exchange reactions by means of nuclear magnetic double resonance. J Chem Phys 1963;39:2892-901.

44. Zhang S, Winter $\mathrm{P}$, Wu K, Sherry A. A novel europium(III)-based MRI contrast agent. J Am Chem Soc. 2001;123:1517-8.

45. Ratnakar S, Woods M, Lubag A, Kovacst Z, Sherry A. Modulation of water exchange in europium(III) DOTA-tetraamide complexes via electronic substituent effects. J Am Chem Soc. 2008;130:6-7.

46. Zhang S, Malloy C, Sherry A. MRI thermometry based on PARACEST agents. J AM CHEM SOC. 2005;127:17572-3.

47. Zhang S, Merritt M, Woessner D, Lenkinski R, Sherry A. PARACEST agents: Modulating MRI contrast via water proton exchange. Acc Chem Res. 2003;36:783-90.

48. Zhang S, Wu K, Sherry A. Unusually sharp dependence of water exchange rate versus lanthanide ionic radii for a series of tetraamide complexes. J Am Chem Soc. 2002;124:4226-7.

49. Zhang S, Michaudet L, Burgess S, Sherry A. The amide protons of an ytterbium(III) dota tetraamide complex act as efficient antennae for transfer of magnetization to bulk water. Angew Chem Int Ed Engl. 2002;41:1919-21.

50. Winter P, Cai K, Chen J, Adair C, Kiefer G, Athey P, et al. Targeted PARACEST nanoparticle contrast agent for the detection of fibrin. Magn Res Med. 2006;56:1384-8.

51. Aime S, Castelli D, Lawson D, Terreno E. Gd-loaded liposomes as T-1, susceptibility, and CEST agents, all in one. J Am Chem Soc. 2007;129:2430-1.

52. Aime S, Delli Castelli D, Terreno E. Supramolecular adducts between poly-L-arginine and [Tm(III)dotp]: A route to sensitivity-enhanced magnetic resonance imaging-chemical exchange saturation transfer agents. Angew Chem Ed Int Engl. 2003;42:4527-9.

53. Snoussi K, Bulte J, Gueron M, van Zijl P. Sensitive CEST agents based on nucleic acid imino proton exchange: Detection of poly $(\mathrm{rU})$ and of a dendrimer-poly(rU) model for nucleic acid delivery and pharmacology. Magn Reson Med. 2003;49:998-1005

54. Louie A, Huber M, Ahrens E, Rothbacher U, Moats R, Jacobs R, et al. In vivo visualization of gene expression using magnetic resonance imaging. Nat Biotechnol. 2000;18:321-5.

55. Aime S, Carrera C, Castelli D, Crich S, Terreno E. Tunable imaging of cells labeled with MRI-PARACEST agents. Angew Chem Int Ed Engl. 2005;44:1813-5.

56. Srinivas M, Morel P, Ernst L, Laidlaw D, Ahrens E. Fluorine-19 MRI for visualization and quantification of cell migration in a diabetes model. Magn Reson Med. 2007;58:725-34.
57. Jiang Z, Liu X, Jeong E, Yu Y. Symmetry-Guided Design and Fluorous Synthesis of a Stable and Rapidly Excreted Imaging Tracer for F-19 MRI. Angew Chem Int Ed Engl. 2009;48:4755-8.

58. Chambers J, Hill P, Aaron J, Han Z, Christianson D, Kuzma N, et al. Cryptophane xenon-129 nuclear magnetic resonance biosensors targeting human carbonic anhydrase. J Am Chem Soc. 2009;131:563-9.

59. Hilty C, Lowery T, Wemmer D, Pines A. Spectrally resolved magnetic resonance imaging of a xenon biosensor. Angew Chem Int Ed Engl. 2006;45:70-3.

60. Goldman M, Johannesson H. Conversion of a proton pair para order into C-13 polarization by RF irradiation, for use in MRI. Comptes Rendus Physique. 2005;6:575-81.

61. Ni Y. Metalloporphyrins and functional analogues as MRI contrast agents. Curr Med Imaging Rev. 2008;4:96-112.

62. Frullano L, Rohovec J, Aime S, Maschmeyer T, Prata M, de Lima J, et al. Towards targeted MRI: New MRI contrast agents for sialic acid detection. Chem-Eur J. 2004;10:5205-17.

63. Laurent S, Vander Elst L, Fu Y, Muller R. Synthesis and physicochemical characterization of Gd-DTPA-B $(\operatorname{sLe}(\mathrm{x})) \mathrm{A}$, a new MRI contrast agent targeted to inflammation. Bioconjug Chem. 2004;15:99-103.

64. Toth E, Helm L, Merbach A. Relaxivity of MRI contrast agents. Top Curr Chem. 2002;221:61-101.

65. Alghisi G, Ruegg C. Vascular integrins in tumor angiogenesis: Mediators and therapeutic targets. Endothelium-J Endoth. 2006;13:113-35.

66. Ruoslahti E. Integrins as signaling molecules and targets for tumor therapy. Kidney Int. 1997;51:1413-7.

67. Stuve O, Gold R, Chan A, Mix E, Zettl U, Kieseier B. a4-integrin antagonism with natalizumab Effects and adverse effects. J Neurol. 2008;255:58-65.

68. Engelhardt B, Kappos L. Natalizumab: Targeting alpha(4)-integrins in multiple sclerosis. NEURODEGENER DIS. 2008;5:16-22.

69. Ruoslahti E. RGD and other recognition sequences for integrins. Annu Rev Cell Dev Biol. 1996;12:697-715.

70. Liu Z, Niu G, Wang F, Chen X. Ga-68-labeled NOTA-RGD-BBN peptide for dual integrin and GRPR-targeted tumor imaging. Eur J Nucl med Mol Imaging. 2009;36:1483-94.

71. Dubey P, Mishra V, Jain S, Mahor S, Vyas S. Liposomes modified with cyclic RGD peptide for tumor targeting. J Drug Target. 2004;12:257-64.

72. Burtea C, Laurent S, Murariu O, Rattat D, Toubeau G, Verbruggen A, et al. Molecular imaging of av $\beta 3$ integrin expression in atherosclerotic plaques with a mimetic of RGD peptide grafted to Gd-DTPA. Cardiovasc Res. 2008;78:148-57.

73. Kreppel F, Kochanek S. Modification of adenovirus gene transfer vectors with synthetic polymers: A scientific review and technical guide. Mol Ther. 2008;16:16-29.

74. Xu H, Regino C, Koyama Y, Hama Y, Gunn A, Bernardo M, et al. Preparation and preliminary evaluation of a biotin-targeted, lectin-targeted dendrimer-based probe for dual-modality magnetic resonance and fluorescence Imaging. Bioconjug Chem. 2007;18:1474-82.

75. Green N. Avidin and Streptavidin. METHOD ENZYMOL. 1990;184:51-67.

76. Debbage P, Jaschke W. Molecular imaging with nanoparticles: giant roles for dwarf actors. Histochem Cell Biol. 2008;130:845-75.

77. Park J, Lee J, Jung J, Yu D, Oh C, Ha S, et al. Gd-DOTA conjugate of RGD as a potential tumor-targeting MRI contrast agent. Chembiochem 2008;9:2811-3.

78. Lauffer R. Paramagnetic metal-complexes as water proton relaxation agents for NMR imaging - theory and design. Chem Rev. 1987;87:901-27. 
79. Zarabi B, Borgman M, Zhuo J, Gullapalli R, Ghandehari H. Noninvasive monitoring of HPMA copolymer-RGDfK conjugates by magnetic resonance imaging. Pharm Res-Dord. 2009;26:1121-9.

80. Princl LEJ, Streba O, Kopeek J. New types of synthetic infusion solutions. III. Elimination and retention of poly-[N-(2-hydroxypropyl)methacrylamide] in a test organism. J Biomed Mater. 1976;10:953-63.

81. Rihova B, Strohalm J, Kubackova K, Jelinkova M, Rozprimova L, Sirova M, et al. Drug-HPMA-HuIg conjugates effective against human solid cancer. Adv Exp Med Biol. 2003;519:125-43.

82. Caiolfa VRZ M, Fiorino A, Frigerio E, et al. Polymer-bound camptothecin: initial biodistribution and antitumour activity studies. J Control Release. 2000;65:105-19.

83. Terwogt J, Huinink W, Schellens J, Schot M, Mandjes I, Zurlo $\mathrm{M}$, et al. Phase I clinical and pharmacokinetic study of PNU166945, a novel water-soluble polymer-conjugated prodrug of paclitaxel. Anticancer Drugs. 2001;12:315-23.

84. Gianasi E, Buckley R, Latigo J, Wasil M, Duncan R. HPMA copolymers platinates containing dicarboxylato ligands. Preparation, characterisation and in vitro and in vivo evaluation. J Drug Target. 2002;10:549-56.

85. Ke T, Jeong E, Wang X, Feng Y, Parker D, Lu Z. RGD targeted poly(L-glutamic acid)-cystamine-(Gd-DO3A) conjugate for detecting angiogenesis biomarker av $\beta 3$ integrin with MRT1 mapping. Int J Nanomed. 2007;2:191-9.

86. Shukla R, Thomas T, Peters J, Kotlyar A, Myc A, Baker J. Tumor angiogenic vasculature targeting with PAMAM dendrimer-RGD conjugates. Chem Comm (Camb). 2005;:5739-41.

87. Boswell C, Eck P, Regino C, Bernardo M, Wong K, Milenic D, et al. Synthesis, characterization, and biological evaluation of integrin av $\beta 3$-targeted PAMAM dendrimers. Mol Pharmaceut. 2008:5:527-39.

88. Peer D, Karp J, Hong S, FaroKHzad O, Margalit R, Langer R. Nanocarriers as an emerging platform for cancer therapy. Nat Nanotechnol. 2007;2:751-60.

89. Unger E, Winokur T, Macdougall P, Rosenblum J, Clair M, Gatenby R, et al. Hepatic metastases -liposomal Gd-DTPA-enhanced MR imaging. Radiology 1989;171:81-5.

90. Magin R, Wright S, Niesman M, Chan H, Swartz H. Liposome delivery of NMR contrast agents for improved tissue imaging. Magn Reson Med. 1986;3:440-7.

91. Fossheim S, Il'yasov K, Hennig J, Bjornerud A. Thermosensitive paramagnetic liposomes for temperature control during MR imaging-guided hyperthermia: In vitro feasibility studies. Acad Radiol. 2000;7:1107-15.

92. Fossheim S, Fahlvik A, Klaveness J, Muller R. Paramagnetic liposomes as MRI contrast agents: Influence of liposomal physicochemical properties on the in vitro relaxivity. Magn Reson Med. 1999;17:83-9.

93. Heverhagen J, Graser A, Fahr A, Muller R, Alfke H. Encapsulation of Gadobutrol in AVE-based liposomal carriers for MR detectability. Magn Reson Imaging. 2004;22:483-7.

94. Sipkins D, Cheresh D, Kazemi M, Nevin L, Bednarski M, Li K. Detection of tumor angiogenesis in vivo by av $\beta 3$-targeted magnetic resonance imaging. Nat Med. 1998;4:623-6.

95. Mulder W, Strijkers G, Habets J, Bleeker E, van der Schaft D, Storm G, et al. MR molecular imaging and fluorescence microscopy for identification of activated tumor endothelium using a bimodal lipidic nanoparticle. FASEB J. 2005;19:2008.

96. Mulder W, Castermans K, van Beijnum J, Egbrink M, Chin P, Fayad Z, et al. Molecular imaging of tumor angiogenesis using av $\beta 3$-integrin targeted multimodal quantum dots. Angiogenesis. 2009;12:17-24.
97. Xie J, Chen K, Lee H, Xu C, Hsu A, Peng S, et al. Ultrasmall $\mathrm{c}(\mathrm{RGDyK})$-coated $\mathrm{Fe}_{3} \mathrm{O}_{4}$ nanoparticles and their specific targeting to integrin av $\beta 3$-rich tumor cells. J Am Chem Soc. 2008;130:7542-3.

98. Lee HY, Li Z, Chen K, Hsu AR, Xu C, Xie J, et al. PET/MRI dual-modality tumor imaging using arginine-glycine-aspartic (RGD)-conjugated radiolabeled iron oxide nanoparticles. J Nucl Med. 2008;49:1371-9.

99. Chen K, Xie J, Xu H, Behera D, Michalski MH, Biswal S, et al. Triblock copolymer coated iron oxide nanoparticle conjugate for tumor integrin targeting. Biomaterials. 2009;30:6912-9.

100.Khemtong C, Kessinger C, Ren J, Bey E, Yang S, Guthi J, et al. In vivo Off-Resonance Saturation Magnetic Resonance Imaging of av $\beta 3$-Targeted Superparamagnetic Nanoparticies. Cancer Res. 2009:69:1651-8.

101.Zurkiya $\mathrm{O}, \mathrm{Hu}$ X. Off-resonance saturation as a means of generating contrast with superparamagnetic nanoparticles. Magn Reson Med. 2006;56:726-32. 